\title{
Overcoming decoherence of Schrödinger cat states formed in a cavity using squeezed-state inputs
}

\author{
R. Y. Teh, P. D. Drummond, and M. D. Reid \\ Centre for Quantum Technology Theory, Swinburne University of Technology, Melbourne 3122, Australia
}

(Received 31 May 2020; accepted 20 October 2020; published 17 December 2020)

\begin{abstract}
A cat state is a superposition of two coherent states with amplitudes $\alpha_{0}$ and $-\alpha_{0}$. Recent experiments create cat states in a microwave cavity field using superconducting circuits. As with degenerate parametric oscillation (DPO) in an adiabatic and highly nonlinear limit, the states are formed in a signal cavity mode via a two-photon dissipative process induced by the down conversion of a pump field to generate pairs of signal photons. The damping of the signal and the presence of thermal fluctuations rapidly decoheres the state, and the effect on the dynamics is to either destroy the possibility of a cat state or else to sharply reduce the lifetime and size of the cat states that can be formed. In this paper, we study the effect on both the DPO and microwave systems of a squeezed reservoir coupled to the cavity. While the threshold nonlinearity is not altered, we show that the use of squeezed states significantly lengthens the lifetime of the cat states. This improves the feasibility of generating cat states of large amplitudes and with a greater degree of quantum macroscopic coherence, which is necessary for many quantum technology applications. Using current experimental parameters for the microwave setup, which requires a modified Hamiltonian, we further demonstrate how squeezed states enhance the quality of the cat states that could be formed in this regime. Squeezing also combats the significant decoherence due to thermal noise, which is relevant for microwave fields at finite temperature. By modeling a thermal squeezed reservoir, we show that the thermal decoherence of the dynamical cat states can be inhibited by a careful control of the squeezing of the reservoir. To signify the quality of the cat state, we consider different signatures including fringes and negativity, and the $C_{l_{1}}$ measure of quantum coherence.
\end{abstract}

DOI: 10.1103/PhysRevResearch.2.043387

\section{INTRODUCTION}

Schrödinger raised the question of how to interpret a macroscopic quantum superposition state in his essay of 1935 [1]. In his analysis, a macroscopic object (a cat) becomes entangled with a microscopic system, creating a paradoxical state that would seem to defy some type of macroscopic reality. The paradoxical state is a superposition of two macroscopically distinguishable states, like a cat being dead or alive. The essay has motivated many papers, including Refs. [2-15] and those that develop decoherence theories to eliminate the possibility of such superposition states forming for massive objects [16].

It remains a challenge to generate a macroscopic superposition where the two states involved are truly macroscopically different. According to quantum mechanics, the coupling between the system and its environment tends to destroy the quantum coherence of the superposition state, particularly as the two states involved in the superposition become macroscopically distinct $[2,9,17]$. Quantum mechanics, however, predicts the existence of macroscopic superposition

Published by the American Physical Society under the terms of the Creative Commons Attribution 4.0 International license. Further distribution of this work must maintain attribution to the author(s) and the published article's title, journal citation, and DOI. states, of arbitrary size, in the absence of decoherence. A fundamental question is whether macroscopic quantum superpositions states can actually exist, or whether quantum mechanics needs modification. This provides motivation to generate superposition states of a larger size and to test the predictions of quantum mechanics in the presence of decoherence.

Cat states based on coherent states are promising for such studies, as well as for applications in quantum information science [18-20]. A cat state is a quantum superposition of two single-mode coherent states with amplitudes $\alpha_{0}$ and $-\alpha_{0}$, which become widely separated in phase space as $\alpha_{0} \rightarrow \infty$. Recent experiments are successful at creating such states in a microwave cavity using superconducting circuits to enhance the nonlinearity. Cat states with $\alpha_{0} \sim 10$ and a high measure of quantum coherence have been generated in these experiments $[6,7,19]$. The coupling of the system to the environment induces decoherence, which destroys the superposition nature of the cat state as the separation in phase space of the two coherent states becomes larger. Decoherence arises as a result of photon loss from the cavity mode [21-25], and from thermal noise which is relevant at microwave frequencies even at low temperatures [15,26-33].

In this paper, we demonstrate how squeezed states may enhance the formation of cat states by modifying the decoherence. Squeezed states are single-mode states of a quantum field that have a variance in one quadrature phase amplitude reduced below the standard quantum limit [34]. Such states 
have been extensively studied [35-44] and created experimentally at optical frequencies [45]. Caves proposed squeezed states as a means to control the vacuum fluctuations entering the input port of the LIGO interferometer, so greater sensitivities for detecting gravitational waves could be achieved [35]. This has recently been implemented [46], and there are numerous other potential applications (including Ref. [47]).

Meccozi and Tombesi [47,48] and Kennedy and Walls [26] suggested using squeezed states to engineer the environment coupled to a macroscopic superposition state, and showed that squeezing in an optimally selected quadrature can suppress the decoherence that otherwise destroys the cat state. This was further explored by Munro and Reid [49], who studied the effect of a squeezed reservoir on the dynamical creation of cat states generated in a highly nonlinear degenerate parametric oscillator (DPO). That treatment, however, did not consider cat states with amplitudes $\alpha_{0}>10$ and was limited to a model applicable to optical rather than microwave systems.

We study the dynamical formation of cat states in a more general description of a DPO and show that the use of squeezed states can significantly increase the lifetime of the cat state in the presence of decoherence. By including an extra Kerr term in the Hamiltonian for the DPO, we are able to model the current microwave experiments, thus providing full solutions showing generation of cat states with $\alpha_{0} \sim 2$ in both the optical and microwave regime. Our conclusions are that the Wigner negativity can be enhanced by a factor of order 2 for parameters corresponding to current microwave experiments. While decoherence can be slowed, squeezed inputs have little effect on the threshold nonlinearity required for the cat state to form.

The study of the dynamics of the formation of cat states is motivated by potential application in quantum information [18] and also by the development of the coherent Ising machine (CIM), an optimization device capable of solving NP-hard problems [50]. The CIM is based on a network of DPOs which currently operate mainly in an optical regime where the reduced nonlinearity makes formation of cat states difficult. However, cat-state regimes may offer advantages due to the enhancement of quantum coherence. Recent proposals [51] and experiments [52] exist for generating cat states in a DPO, which may be applied to a DPO network for adiabatic quantum computation [53] to solve these NP-hard problems. The application of squeezed states to the CIM has been more recently studied and shown to potentially enhance performance [54].

Cat states are predicted to be created in the signal cavity mode of the DPO through a mechanism based on a twophoton dissipative process $[19,21,23,24]$. The two-photon process originates from the parametric interaction in which pump photons are down-converted to correlated pairs of signal photons [55], and can be realized in both optical and microwave cavities. However, the presence of signal losses significantly alters the dynamics, so a cat state is not generated unless there is a very strong parametric nonlinearity $[21,25,49,56-59]$. While not yet feasible for optical modes, this can be achieved for microwave superconducting circuit experiments, where the cat states are generated as a transient state $[19,59]$. In this paper, we study the dynamics of the cat states and their decoherence as the signal losses are increased.
With squeezed input states into the cavity, we then explain that although the threshold nonlinearity is not reduced, decoherence can be compensated for to allow cat states of longer lifetimes and with larger amplitudes $\alpha_{0}$ to be formed, which is relevant to the resource theory of non-Gaussianity [60].

Thermal noise also destroys the cat state, and we therefore investigate the effect of squeezed thermal reservoirs on the feasibility of creating the cat states. We model the squeezed inputs for temperatures corresponding to the microwave experiment of Leghtas et al. [19]. We allow for both a thermalized squeezed reservoir, where the cavity evolves coupled to a finite-temperature environment [61], and also the input of a squeezed thermal state [62], where the squeezed state is created using a finite-temperature source [40]. We show that an optimal amount of squeezing will enhance the cat state that is formed. Our work complements previous treatments by Kennedy and Walls [26], Serafini et al. [29], and Bennett and Bowen [33], who either analyze the effect of squeezing applied to a cat state generated in an optomechanical cavity mode, as in Ref. [63] or else examine cat states coupled to thermal reservoirs where a squeezed thermal state is injected. Other mechanisms have also been proposed to enhance the lifetime of the cat state in a microwave cavity [64].

For any experimental situation, the cat states that are generated are not pure states. This is particularly relevant for the cat states generated in a cavity where signal loss is important. As such, it is necessary to carefully signify the existence and quality of the cat states [65]. In this paper, we certify cat states using three different criteria: We use interference fringes $[4,9]$, the negativity of the Wigner function [66], and the $C_{l_{1}}$ measure of quantum coherence [67].

\section{HAMILTONIAN AND MASTER EQUATION WITH A SQUEEZED RESERVOIR}

We begin by reviewing a model of the DPO and the master equation formalism whereby the interaction between the system and the external environment can be taken into account. The degenerate parametric oscillator has two modes, the pump mode and signal mode with a frequency of $2 \omega_{0}$ and $\omega_{0}$, respectively. These modes are resonant in a cavity. An external pumping field at frequency $2 \omega_{0}$ creates photons in the pump mode, which in turn interacts with a nonlinear crystal that generates correlated pairs of photons in the signal mode at half the frequency of the pump mode.

There is single-photon damping in both the signal and pump modes with rates $\gamma_{1}$ and $\gamma_{2}$ respectively. These damping rates model the leakage of photons out of the cavity. We assume this takes place through an end mirror of the cavity. We consider a single-sided cavity for the signal mode, where one mirror is totally reflecting, thus not allowing signal photons to leak through. Typically, the pump mode decay rate is much larger than the signal mode decay rate $\left(\gamma_{2} \gg \gamma_{1}\right)$, which allows the pump mode to be adiabatically eliminated. There is also a two-photon loss process due to the conversion of two signal mode photons back to a single pump mode photon.

The interaction Hamiltonian that captures all these effects, after carrying out the adiabatic elimination procedure, and 
also including a Kerr effect, is given by $[55,57,58,68]$

$$
\begin{aligned}
H / \hbar= & i\left(\frac{\bar{g} \epsilon}{\gamma_{2}} a_{1}^{\dagger 2}-\frac{\bar{g}^{*} \epsilon^{*}}{\gamma_{2}} a_{1}^{2}\right)+\bar{\chi} a_{1}^{\dagger 2} a_{1}^{2} \\
& +a_{1}^{\dagger} \Gamma+a_{1} \Gamma^{\dagger}+\frac{|\bar{g}|^{2}}{4 \gamma_{2}}\left(a_{1}^{2} \Gamma_{2}^{\dagger}+a_{1}^{\dagger 2} \Gamma_{2}\right) .
\end{aligned}
$$

Here, $\bar{g}$ is the parametric coupling strength between the pump and signal, and $\bar{\chi}$ is an anharmonic Kerr effect nonlinearity in the signal mode. The terms $\Gamma$ and $\Gamma_{2}$ are operators for the external reservoirs that give rise to single-photon and two-photon loss processes respectively, which also allows finite-temperature effects to be included.

Next, we discuss the nature of the environment that interacts with the signal mode. This can be controlled via the input field to the single end-mirror of the cavity, which allows leakage of signal cavity photons into the external environment and can be formalized using input-output cavity theory $[37,69]$. The signal reservoir $\Gamma$ can be expanded in terms of external boson reservoir operators $\hat{b}^{\dagger}(\omega)$. These correspond to a continuum of traveling wave modes, each of which is a combination of the modes input to the cavity and output from the cavity. They are expanded in terms of their individual mode frequencies $\omega$ as

$$
\Gamma=\int_{0}^{\infty} d \omega \kappa(\omega) \hat{b}(\omega),
$$

with bosonic commutation relations of

$$
\left[b(\omega), b^{\dagger}\left(\omega^{\prime}\right)\right]=\delta\left(\omega-\omega^{\prime}\right) .
$$

Here $\kappa(\omega)$ is assumed slowly varying near the resonance at $\omega_{0}$, and the cavity amplitude damping rate is $\gamma_{1}=\pi\left|\kappa\left(\omega_{0}\right)\right|^{2}$. We are interested in the incoming field $\Gamma_{\text {in }}$, defined such that, if $b_{0}(\omega)$ is the initial reservoir field at $t_{0}$, then

$$
\Gamma_{\text {in }}=\int_{0}^{\infty} d \omega \kappa(\omega) e^{-i \omega\left(t-t_{0}\right)} b_{0}(\omega) .
$$

For an initial time $t_{0} \rightarrow-\infty$, the input state of the reservoir is assumed to be such that, in a region of frequency of $\omega \approx \omega_{0}$, which is large compared to the cavity bandwidth $\gamma_{1}$ :

$$
\begin{aligned}
\left\langle b^{\dagger}\left(\omega, t_{0}\right) b\left(\omega^{\prime}, t_{0}\right)\right\rangle & =N \delta\left(\omega-\omega^{\prime}\right), \\
\left\langle b\left(\omega, t_{0}\right) b^{\dagger}\left(\omega^{\prime}, t_{0}\right)\right\rangle & =(N+1) \delta\left(\omega-\omega^{\prime}\right), \\
\left\langle b\left(\omega, t_{0}\right) b\left(\omega^{\prime}, t_{0}\right)\right\rangle & =M e^{-2 i \omega t} \delta\left(\omega+\omega^{\prime}-2 \omega_{0}\right), \\
\left\langle b^{\dagger}\left(\omega, t_{0}\right) b^{\dagger}\left(\omega^{\prime}, t_{0}\right)\right\rangle & =M^{*} e^{2 i \omega t} \delta\left(\omega+\omega^{\prime}-2 \omega_{0}\right) .
\end{aligned}
$$

As shown by Gardiner and Collett [69] and elsewhere $[26,49,70]$, a bath with quantum fluctuations corresponding to a multimode squeezed state near the resonant frequency $\omega_{0}$ has the following input moments before turning on the bath coupling, for $t, t^{\prime} \rightarrow-\infty$ :

$$
\begin{aligned}
& \left\langle\Gamma_{\mathrm{in}}^{\dagger}(t) \Gamma_{\mathrm{in}}\left(t^{\prime}\right)\right\rangle=2 \gamma_{1} N \delta\left(t-t^{\prime}\right), \\
& \left\langle\Gamma_{\mathrm{in}}(t) \Gamma_{\mathrm{in}}^{\dagger}\left(t^{\prime}\right)\right\rangle=2 \gamma_{1}(N+1) \delta\left(t-t^{\prime}\right), \\
& \left\langle\Gamma_{\mathrm{in}}(t) \Gamma_{\mathrm{in}}\left(t^{\prime}\right)\right\rangle=2 \gamma_{1} M e^{-2 i \omega_{0} t} \delta\left(t-t^{\prime}\right), \\
& \left\langle\Gamma_{\mathrm{in}}^{\dagger}(t) \Gamma_{\mathrm{in}}^{\dagger}\left(t^{\prime}\right)\right\rangle=2 \gamma_{1} M^{*} e^{2 i \omega_{0} t} \delta\left(t-t^{\prime}\right) .
\end{aligned}
$$

Here $N$ is a positive, real number characterizing the mean photon number of the state of the environment and $M=$
$|M| e^{i \Phi}$ is a complex number that characterizes the nature of the squeezing. When $M=0$, the bath fluctuations are not squeezed. When $M \neq 0$ at zero temperature, the reservoir is in a squeezed state centered around the frequency $\omega$ and $N$ is the mean photon number of the corresponding squeezed state. The link between $N$ and $M$ depends on the precise model for the squeezed reservoir that we adopt. The results of Eqs. (6) can be found in Eq. (5) of Ref. [49] and Eqs. (4.26) and (4.27) in Ref. [69].

Thermal noise is a secondary source of decoherence known to destroy the quantum coherence of cat states [26]. In this paper, we mainly focus on the simple case where a squeezed vacuum is applied to the system at zero temperature, in which case $N$ will be zero if there is no squeezing of the reservoir fluctuations. The zero temperature limit is justified for optical frequencies where thermal noise is low even at room temperature, and for microwave systems it is achievable by cooling.

However, to gain some insight into the sensitivity of the quantum coherences on temperature, our equations can allow for thermal noise, which may be modeled in two ways. We may consider the reservoir to be a thermalized squeezed state or else a squeezed thermal state $[61,62]$. The statistics arising in each case, and the resulting effects on the decoherence of an ideal cat state, are summarized in the Appendix. In both cases, the reservoir has a mean photon number $N=N_{\text {th }}+N_{s}$ that is a sum of a number $N_{\text {th }}$ due to finite temperature, and the photon number due to the squeezed state $N_{s}$. The corresponding statistical moments can be expressed in the form given by Eqs. (6). However, for a squeezed thermal state (defined by a squeezing interaction acting on a system prepared initially in a thermal state), the mean photon number contains a cross term between the squeezing and thermal noise contribution [61], which leads to a stronger reduction in quantum noise. We note that while thermal noise is significant at microwave frequencies, squeezing at this frequency has been achieved experimentally [40,42-44,71]. The states generated at a finite temperature by some squeezing mechanism would be modeled by a squeezed thermal state.

We may also justify the model of the reservoir for the cavity field where $N_{\text {th }}$ is due to the temperature $T$ of the environment of the cavity as the cat states evolve. Here we suppose the squeezed fields to be generated at zero temperature, so the thermal noise $N_{\text {th }}$ term is independent of the characterization of the squeezing that enters the cavity. This model corresponds to a thermalized squeezed reservoir.

We need to know how $N$ and $M$ relate to the amount of squeezing of the reservoir input. For a squeezed state produced at zero temperature from an optical parametric oscillator, we have $N_{\text {th }}=0$, and the values of $N_{s}$ and $M$ are linked, the degree of squeezing being determined by $N$. In particular, it is known that the optimal squeezing produced from an optical parametric oscillator at zero temperature is achieved when $|M|=\sqrt{N_{s}\left(N_{s}+1\right)}[26,37,47,49,69]$. To this end, we relate the amount of squeezing in the quadrature to the parameters $N_{s}$ and $M$. Recall that the bath which interacts with the signal mode is modeled by a harmonic oscillator satisfying a set of noise correlations as given in Eqs. (6). Defining the 
general rotated $X_{\theta}$ and $P_{\theta}$ quadratures for this bath mode as

$$
\begin{aligned}
& X_{\theta} \equiv \frac{1}{\sqrt{2}}\left(\Gamma_{\text {in }} \mathrm{e}^{-i \theta}+\Gamma_{\text {in }}^{\dagger} \mathrm{e}^{i \theta}\right), \\
& P_{\theta} \equiv \frac{1}{i \sqrt{2}}\left(\Gamma_{\text {in }} \mathrm{e}^{-i \theta}-\Gamma_{\text {in }}^{\dagger} \mathrm{e}^{i \theta}\right),
\end{aligned}
$$

we calculate the variances of $X_{\theta}$ and $P_{\theta}$ with squeezing, with respect to their corresponding variances in $X_{\theta}$ and $P_{\theta}$ of a vacuum state. They are, using the statistical moments in Eqs. (6), given by

$$
\begin{aligned}
\Delta^{2} X_{\theta} & =\left\langle\Gamma_{\text {in }}^{\dagger} \Gamma_{\text {in }}\right\rangle+1+\frac{1}{2}\left\langle\Gamma_{\text {in }}^{2}\right\rangle e^{-2 i \theta}+\frac{1}{2}\left\langle\Gamma_{\text {in }}^{\dagger 2}\right\rangle e^{2 i \theta} \\
& =2 N_{s}+1+|M|\left[e^{i(\Phi-2 \theta)}+e^{-i(\Phi-2 \theta)}\right]
\end{aligned}
$$

and

$$
\begin{aligned}
\Delta^{2} P_{\theta} & =\left\langle\Gamma_{\text {in }}^{\dagger} \Gamma_{\text {in }}\right\rangle+1-\frac{1}{2}\left\langle\Gamma_{\text {in }}^{2}\right\rangle e^{-2 i \theta}-\frac{1}{2}\left\langle\Gamma_{\text {in }}^{\dagger 2}\right\rangle e^{2 i \theta} \\
& =2 N_{s}+1-|M|\left[e^{i(\Phi-2 \theta)}+e^{-i(\Phi-2 \theta)}\right],
\end{aligned}
$$

where $M=|M| e^{i \Phi}$ as previously defined. Choosing $M$ so $\Phi=2 \theta$ and $|M|=\sqrt{N_{s}\left(N_{s}+1\right)}$, in the limit of large $N_{s}$, the variance of $X_{\theta=0}=X$ is

$$
\Delta^{2} X \sim 4\left(N_{s}+\frac{1}{2}\right)
$$

and the variance of $P_{\theta=0}=P$ is

$$
\Delta^{2} P \sim \frac{1}{4 N_{s}}
$$

The fluctuations in the $P$ quadrature can be negligibly small in this limit. From Eq. (9), squeezing in the quadrature $P_{\theta}$ is obtained when $\Phi$ is chosen such that $\Phi=2 \theta$. We will consider here $\theta=0$, so $M$ is real and positive.

The full dynamics, including all the effects discussed in previous paragraphs, is dictated by the master equation in the Markovian approximation, as given below:

$$
\begin{aligned}
\frac{\partial}{\partial t} \rho= & \frac{|\bar{g} \epsilon|}{2 \gamma_{2}}\left[a^{\dagger 2}-a^{2}, \rho\right]+\frac{1}{2}\left(\frac{\bar{g}^{2}}{2 \gamma_{2}}\right)\left(2 a^{2} \rho a^{\dagger 2}-a^{\dagger 2} a^{2} \rho-\rho a^{\dagger 2} a^{2}\right)-i \frac{\bar{\chi}}{2}\left[a^{\dagger 2} a^{2}, \rho\right] \\
& +\left(N_{\text {th }}+N_{s}+1\right) \gamma_{1}\left[2 a \rho a^{\dagger}-a^{\dagger} a \rho-\rho a^{\dagger} a\right]+\left(N_{\text {th }}+N_{s}\right) \gamma_{1}\left[2 a^{\dagger} \rho a-a a^{\dagger} \rho-\rho a a^{\dagger}\right] \\
& -M \gamma_{1}[2 a \rho a-a a \rho-\rho a a]-M^{*} \gamma_{1}\left[2 a^{\dagger} \rho a^{\dagger}-a^{\dagger} a^{\dagger} \rho-\rho a^{\dagger} a^{\dagger}\right] .
\end{aligned}
$$

With no loss of generality, we can choose the phase of $\bar{g}$ such that $\bar{g} \epsilon=\bar{g}^{*} \epsilon^{*}[25,72]$. Here, $\rho$ is the density operator of the signal mode. It is convenient to scale all parameters with respect to the signal mode single-photon decay rate $\gamma_{1}$. This defines a dimensionless time $\tau=\gamma_{1} t$; a dimensionless pump strength $\lambda=|\bar{g} \epsilon| /\left(\gamma_{1} \gamma_{2}\right)$, a dimensionless effective parametric interaction $g=\sqrt{\bar{g}^{2} /\left(2 \gamma_{1} \gamma_{2}\right)}$ and a dimensionless Kerr strength $\chi^{\prime}=\bar{\chi} / \gamma_{1}$.

In the presence of damping the master equation, Eq. (12), is best solved numerically. In this paper, the density op- erator is expanded in the number state basis $\{|n\rangle\}$ with a suitable photon number cutoff, which allows cat-state signatures to be computed. With the dimensionless parameters, the master equation, Eq. (12), in the number state basis is reduced to a set of partial differential equations of the form

$$
\frac{\partial}{\partial \tau} \rho_{n, m}=\sum_{i} \sum_{j} \mathcal{L}_{n m}^{i j} \rho_{i, j},
$$

where

$$
\begin{aligned}
\mathcal{L}_{n m}^{i j}= & \frac{\lambda}{2} \sqrt{n(n-1)} \delta_{n-2}^{i} \delta_{m}^{j}+\frac{\lambda}{2} \sqrt{m(m-1)} \delta_{n}^{i} \delta_{m-2}^{j}-\frac{\lambda}{2} \sqrt{(n+1)(n+2)} \delta_{n+2}^{i} \delta_{m}^{j}-\frac{\lambda}{2} \sqrt{(m+1)(m+2)} \delta_{n}^{i} \delta_{m+2}^{j} \\
& -i \frac{\chi^{\prime}}{2}[n(n-1)-m(m-1)] \delta_{n}^{i} \delta_{m}^{j}+g^{2} \sqrt{(n+1)(n+2)(m+1)(m+2)} \delta_{n+2}^{i} \delta_{m+2}^{j}-\frac{g^{2}}{2}[n(n-1)+m(m-1)] \delta_{n}^{i} \delta_{m}^{j} \\
& +\left(N_{\mathrm{th}}+N_{s}+1\right)\left[2 \sqrt{(n+1)(m+1)} \delta_{n+1}^{i} \delta_{m+1}^{j}-n \delta_{n}^{i} \delta_{m}^{j}-m \delta_{n}^{i} \delta_{m}^{j}\right] \\
& +\left(N_{\mathrm{th}}+N_{s}\right)\left[2 \sqrt{n m} \delta_{n-1}^{i} \delta_{m-1}^{j}-(n+1) \delta_{n}^{i} \delta_{m}^{j}-(m+1) \delta_{n}^{i} \delta_{m}^{j}\right] \\
& -M\left[2 \sqrt{(n+1) m} \delta_{n+1}^{i} \delta_{m-1}^{j}+\sqrt{(n+1)(n+2)} \delta_{n+2}^{i} \delta_{m}^{j}+\sqrt{m(m-1)} \delta_{n}^{i} \delta_{m-2}^{j}\right] \\
& -M^{*}\left[2 \sqrt{n(m+1)} \delta_{n-1}^{i} \delta_{m+1}^{j}+\sqrt{n(n-1)} \delta_{n-2}^{i} \delta_{m}^{j}+\sqrt{(m+1)(m+2)} \delta_{n}^{i} \delta_{m+2}^{j}\right]
\end{aligned}
$$

Here, $\delta_{n}^{i}$ is a Kronecker delta function with $\delta_{n}^{i}=1$ if $i=n$ and $\delta_{n}^{i}=0$ otherwise.

Note that in the absence of single-photon damping $\left(\gamma_{1}=0\right)$, the steady-state solution for the master equation Eq. (12) for an initial vacuum state corresponds to a cat state

$$
\left|\psi_{c}\right\rangle=\mathcal{N}\left(\left|\alpha_{0}\right\rangle+\left|-\alpha_{0}\right\rangle\right),
$$

where $\quad \alpha_{0}=\sqrt{\lambda /\left(g^{2}+i \chi^{\prime}\right)}=\sqrt{\lambda / g^{2}(1+i \chi)}, \quad \chi \equiv \chi^{\prime} / g^{2}$ and $\mathcal{N}=\left[2\left(1+e^{-2\left|\alpha_{0}\right|^{2}}\right)\right]^{-1 / 2}$ is the normalization constant. 
This cat state has an even photon number as required for a two-photon process arising from the vacuum [23,24].

\section{CAT-STATE SIGNATURES}

To verify the presence of a cat state, we will compute several different cat-state signatures. The objective is to distinguish the cat state $\left|\psi_{\text {cat }}\right\rangle=\mathcal{N}(|\alpha\rangle+|-\alpha\rangle)$ of Eq. (15) (where $\alpha$ is real and $\mathcal{N}$ is a normalization constant) from a classical mixture $\rho_{\text {mix }}$ of the two coherent states, given as

$$
\rho_{\text {mix }}=\frac{1}{2}(|\alpha\rangle\langle\alpha|+|-\alpha\rangle\langle-\alpha|) .
$$

Alternative approaches to detecting macroscopic coherence are available but are not employed in this paper $[65,73,74]$. These include signatures and measures for macroscopic quantum coherence based on variances and quantum Fisher information.

\section{A. Interference fringes}

A commonly used signature is the presence of interference fringes. Rotated signal mode quadrature operators are defined as $x_{\theta}=\left(e^{-i \theta} a+e^{i \theta} a^{\dagger}\right) / \sqrt{2}$ and $p_{\theta}=\left(e^{-i \theta} a-e^{i \theta} a^{\dagger}\right) / \sqrt{2} i$. In particular, for $\theta=0, x_{\theta}$ and $p_{\theta}$ correspond to the standard $x$ - and $p$-quadrature operators, respectively. The $x_{\theta}$-quadrature probability distribution $P\left(x_{\theta}\right)$ for a density operator $\rho=$ $\sum_{n, m} \rho_{n m}|n\rangle\langle m|$ in the number state basis is given by

$$
\left\langle x_{\theta}|\rho| x_{\theta}\right\rangle=\sum_{n, m} \rho_{n m}\left\langle x_{\theta} \mid n\right\rangle\left\langle m \mid x_{\theta}\right\rangle
$$

where

$$
\left\langle x_{\theta} \mid n\right\rangle=\frac{e^{-i \theta n}}{\sqrt{2^{n} n ! \sqrt{\pi}}} e^{-\frac{x_{\theta}^{2}}{2}} H_{n}\left(x_{\theta}\right) .
$$

Observation of two well-separated Gaussian peaks in $P\left(x_{\theta}\right)$ along with interference fringes in $P\left(p_{\theta}\right)$ gives evidence of the system being in a cat state as opposed to a mixture of the two coherent states.

\section{B. Wigner function negativity}

We also compute the Wigner function and its negativity. It has been shown that the Wigner function in the number state basis has the following expression [75,76]:

$$
W\left(\alpha, \alpha^{*}\right)=e^{-2|\alpha|^{2}} c_{0}+2 \operatorname{Re}\left(e^{-2|\alpha|^{2}} \sum_{l=1}^{N_{c}} c_{l}(2 \alpha)^{l}\right),
$$

where

$$
c_{l}=\sum_{n=0}^{N_{c}-l} \rho_{n, l+n} \frac{2(-1)^{n}}{\pi} \sqrt{\frac{n !}{(l+n) !}} L_{n}^{l}\left(4|\alpha|^{2}\right) .
$$

The corresponding negativity is defined as [66]

$$
\delta=\frac{1}{2} \int\left[\left|W\left(\alpha, \alpha^{*}\right)\right|-W\left(\alpha, \alpha^{*}\right)\right] d^{2} \alpha .
$$

The Wigner negativity quantifies the nonclassicality of a physical state. If the Wigner function is positive, then the Wigner distribution can be interpreted as a probability distribution for variable $x_{\theta}$ and $p_{\theta}$, thus giving an explanation for the observed quadrature distributions $P\left(x_{\theta}\right)$ and $P\left(p_{\theta}\right)$. A nonzero negativity excludes this interpretation $[22,59]$. The negativity Eq. (21) of a pure, even (odd) cat state $W_{+}\left(W_{-}\right)$is calculated from the Wigner function [15,59]:

$$
\begin{aligned}
W_{ \pm}\left(\alpha, \alpha^{*}\right)= & \frac{2}{\pi} \mathcal{N}_{ \pm}^{2}\left\{\exp \left[-2\left(\alpha^{*}-\alpha_{0}^{*}\right)\left(\alpha-\alpha_{0}\right)\right]\right. \\
& +\exp \left[-2\left(\alpha^{*}+\alpha_{0}^{*}\right)\left(\alpha+\alpha_{0}\right)\right] \\
& \pm\left\langle\alpha_{0} \mid-\alpha_{0}\right\rangle \exp \left[-2\left(\alpha^{*}-\alpha_{0}^{*}\right)\left(\alpha+\alpha_{0}\right)\right] \\
& \left. \pm\left\langle-\alpha_{0} \mid \alpha_{0}\right\rangle \exp \left[-2\left(\alpha^{*}+\alpha_{0}^{*}\right)\left(\alpha-\alpha_{0}\right)\right]\right\}
\end{aligned}
$$

However, we note that it is possible to have a positive Wigner function even when significant quantum coherence exists, as shown in a previous work [15]. Other cat-state signatures have to be inferred to conclusively verify the existence of a catlike state. We emphasize this by further pointing out that an impure cat state or a broader class of mixtures can possess a nonzero Wigner negativity.

\section{Quantum coherence}

With this motivation, we may also use quantum coherence as a signature of the cat state. The quantum coherence is quantified by a non-negative number, assigned as a measure of the degree of coherence. Two particular measures have been developed [67], the relative entropy of coherence and the $l_{1}$ norm of coherence. Here, we consider the $l_{1}$ norm defined as

$$
C_{l_{1}}(\rho)=\sum_{i \neq j} \sum_{j}\left|\rho_{i j}\right|,
$$

where $\rho$ is the density operator and $|i\rangle$ a basis set. One may evaluate a measure of total quantum coherence, using the $l_{1}$ norm quantum coherence measure defined for continuous variables:

$$
\begin{aligned}
C_{l_{1}}(\rho) & \equiv \int_{-\infty}^{\infty} \int_{-\infty}^{\infty}\left|\rho_{x x^{\prime}}\right| d x d x^{\prime}-\int_{-\infty}^{\infty}\left|\rho_{x x}\right| d x \\
& =\int_{-\infty}^{\infty} \int_{-\infty}^{\infty}\left|\left\langle x|\rho| x^{\prime}\right\rangle\right| d x d x^{\prime}-\int_{-\infty}^{\infty}|\langle x|\rho| x\rangle| d x .
\end{aligned}
$$

Taking $\alpha_{0}$ to be real, we see that for the mixture, $C_{l_{1}}\left(\rho_{\text {mix }}\right)=$ $2 \sqrt{\pi}-1$, while for the cat state Eq. (15), $C_{l_{1}}\left(\rho_{\text {cat }}\right)=$ $8 \sqrt{\pi} \mathcal{N}^{2}-1\left(\right.$ where $\mathcal{N}=\left[2\left(1+e^{-2\left|\alpha_{0}\right|^{2}}\right)\right]^{-1 / 2}$ is the normalization factor previously defined). The quantum coherence for the mixture arises from the quantum coherence of the coherent states $| \pm \alpha\rangle$ involved in the mixture. The quantum coherence for a cat state contains an extra contribution due to the state being a macroscopic superposition.

Quantum coherence does not indicate negativity of the Wigner function and is thus a measure of a different type of nonclassicality. It has been shown that squeezed states possess a high degree of quantum coherence [65,74], yet also possess a positive Wigner function, thus admitting local hidden variable theories for experiments involving quadrature phase amplitude measurements.

\section{Fidelity}

Another quantity that is often used to characterize quantum states is the fidelity between the physical state of the system 
and a specific targeted state. The fidelity is a distance measure that indicates how close two states are [77]. This has been used both in theoretical predictions [78] and in experimental measurements [4,7]. Here, the fidelity is calculated with respect to an even, pure cat state $\left|\psi_{c}\right\rangle=\mathcal{N}\left(\left|\alpha_{0}\right\rangle+\left|-\alpha_{0}\right\rangle\right)$ in Eq. (15). In the following, we express the fidelity where the physical states are expressed in the number state basis:

$$
\begin{aligned}
\mathcal{F}= & \left\langle\psi_{c}|\rho| \psi_{c}\right\rangle \\
= & \mathcal{N}^{2} e^{-\left|\alpha_{0}\right|^{2}}\left[\sum_{n=0}^{\infty} \frac{\left(\alpha_{0}^{*}\right)^{n}+\left(-\alpha_{0}^{*}\right)^{n}}{\sqrt{n !}}\langle n|\right] \\
& \times\left[\sum_{k, l} \rho_{k l}|k\rangle\langle l|\right] \times\left[\sum_{m=0}^{\infty} \frac{\left(\alpha_{0}\right)^{m}+\left(-\alpha_{0}\right)^{m}}{\sqrt{m !}}|m\rangle\right] \\
= & \mathcal{N}^{2} e^{-\left|\alpha_{0}\right|^{2}} \sum_{k, l}\left[\frac{\left(\alpha_{0}^{*}\right)^{k}+\left(-\alpha_{0}^{*}\right)^{k}}{\sqrt{k !}}\right] \\
& \times\left[\sum_{m=0}^{\infty} \frac{\left(\alpha_{0}\right)^{l}+\left(-\alpha_{0}\right)^{l}}{\sqrt{l !}}\right] \rho_{k l} .
\end{aligned}
$$

The fidelity is 1 when the state of the system is a pure, even cat state $\left|\psi_{c}\right\rangle$. For a mixed state $\rho_{\text {mix }}=$ $\left(\left|\alpha_{0}\right\rangle\left\langle\alpha_{0}|+|-\alpha_{0}\right\rangle\left\langle-\alpha_{0}\right|\right) / 2$ in Eq. (16), the fidelity is $\left(1+e^{-2\left|\alpha_{0}\right|^{2}}\right) / 2$ (see Appendix).

We note that while fidelity can certainly indicate if a generated state is close to a target state, it provides no clear indication that a state has any of the characteristic properties of a Schrodinger cat at some threshold fidelity. As a result, this measure is best used in combination with other measures described above that specifically target the catlike nature of the quantum state.

\section{E. Purity and number distribution}

Finally, states created in the presence of signal damping, $\gamma_{1} \neq 0$, will not be pure. A full discussion of this is given in Refs. [21,23-25,58,59]. The degree of purity of the catlike states that are created can be extracted from the measure of purity $\mathcal{P}$ :

$$
\mathcal{P}=\operatorname{Tr}\left(\rho^{2}\right) .
$$

In the limit of long times where there are signal cavity losses, a system initially prepared in the vacuum state will become equivalent to a nearly equal mixture of two coherent states, and the long-time purity of the system then approaches $50 \%$. An indication of the purity of the cat state is also given by the photon number distribution $P(n)$. This distribution $P(n)$ has zero values for odd $n$ for the cat state Eq. (15), which is generated by the parametric process from a vacuum initial state.

\section{DEGENERATE PARAMETRIC OSCILLATION WITH A SQUEEZED RESERVOIR}

\section{A. Squeezed reservoir fields}

From the master equation, Eq. (12), the free parameters are the pump strength $\lambda$, the effective parametric interaction $g$, the Kerr nonlinearity $\chi$, the two parameters that characterize the reservoir, $N$ and $M$, and the dimensionless time $\tau$. In particular, it has been shown, in the limit of zero single-photon damping, that the cat state formed is given as Eq. (15) with $\left|\alpha_{0}\right|=\sqrt{\lambda / g^{2} \sqrt{1+\chi^{2}}}$. For all the different sets of parameters investigated in this paper, we set the initial state of the system to be in the vacuum state. This also implies that the squeezed vacuum input is switched on simultaneously with the pump, or in some cases at a later time.

First, we take $\chi=0$, in which case the amplitude is real. We assume zero temperature, $T=0$, which is a good approximation to the DPO at room temperature for optical frequencies where thermal noise is negligible. Here, we fix $\left|\alpha_{0}\right|=10$ and $g=2.5$, and study the effect of different squeezing strengths at zero temperature $\left(N_{\text {th }}=0\right)$. Figure 1 shows the evolution of the two peaks in the $x$ quadrature, reflecting the possible presence of a cat state. Given that the ideal cat state has a real amplitude, as in Meccozi and Tombesi [47,48], the optimal direction of squeezing to combat the decoherence due to loss is directed along the $P$ axis. This corresponds to the choice that $M$ is real and positive.

Figure 1 shows the time evolution of the $p$-quadrature probability distribution at zero temperature with and without a squeezed input. We see that interference fringes start to appear after $\tau=0.005$. However, for a squeezed input, the interference fringes are more refined. The visibility of the interference fringes is significantly higher than the fringes without a squeezed reservoir at the same dimensionless time $\tau$. In Fig. 1, we see that the $x$-quadrature probability distribution for the cases with and without squeezing are similar. For real $\alpha_{0}$, the existence of two peaks in the $x$ quadrature implies well-separated coherent state amplitudes, while the interference fringes in the corresponding $p$ quadrature are an indication of the quantum nature of the underlying state. These fringes rule out the possibility of the state being in a classical mixture of two coherent states with amplitudes $\pm \alpha_{0}$. Clearly, from the figures, a squeezed input enhances the lifetime of the cat state.

The photon number distribution provides a clear perspective on why the interference fringes have higher visibility for a squeezed input. By comparing the photon number distribution at $\tau=0.0075$ and 0.0150 with squeezing in Figs. 2(b) and 2(d) and without squeezing in Figs. 2(a) and 2(c), we see that the probability for an odd number is suppressed in the presence of a squeezed reservoir. In other words, the system retains a high probability to be in an even cat state for a longer time, as compared to the case without the squeezed reservoir. The photon number distribution results suggest that the effect of squeezing is to maintain the purity of the state for longer times. We quantify this by evaluating the quantum coherence, fidelity, negativity and purity, in Figs. 3, 4, and 5. The quantum coherence, fidelity and Wigner negativity signatures are improved with the increase of squeezing in the reservoir.

The results for purity as in Eq. (26) are plotted in Fig. 5. Larger squeezing in the input reservoir leads to higher purity at a given time. We note the dip in the purity during the time evolution before increasing again. This is also observed in the case where the initial and final states are pure states, implying that the state during evolution is not generally a pure one 
(a)

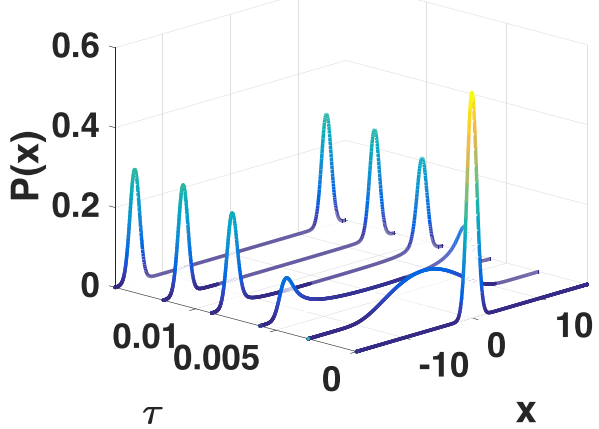

(b)

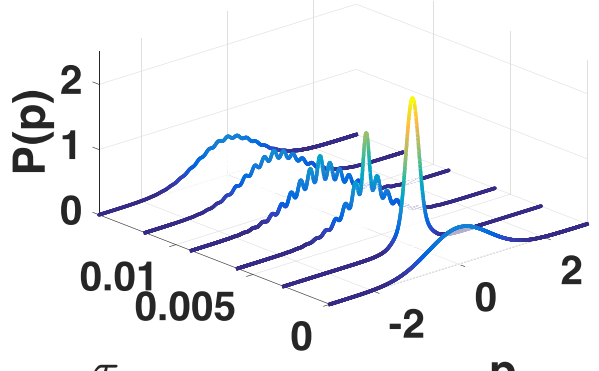

(c)

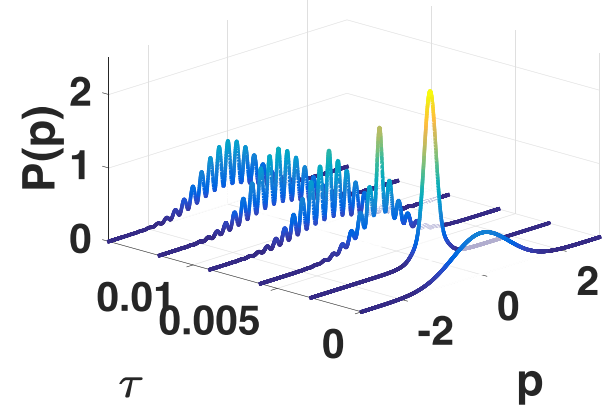

FIG. 1. Effect of squeezed reservoirs on the cat-state formation in a highly nonlinear DPO without Kerr nonlinearity, at zero temperature. The top plot (a) shows the $x$-quadrature probability distribution as a function of time $\tau$. The cases without squeezing $\left(N_{s}=0\right)$ and with squeezing $\left(N_{s}=1, M=\sqrt{2}\right)$ share similar time evolution of the $x$-quadrature probability distribution. The lower plots [(b) and (c)] show the $p$-quadrature probability distribution (b) without squeezing $N_{s}=0$, and (c) with squeezing $N_{s}=1$. The parameters are $g=2.5$, $\alpha_{0}=\lambda / g^{2}=100, N_{\text {th }}=0$, and $M=\sqrt{N_{s}\left(N_{s}+1\right)}$.

[23]. Eventually, for long times, the purity approaches $50 \%$, as the cat state becomes a nearly equal mixture of two coherent states.

\section{B. Large cat amplitudes}

The results presented so far suggest that a fragile mesoscopic (macroscopic) quantum state could well be preserved under the influence of a squeezed reservoir. In the following, we consider a large catlike state with an amplitude $\left|\alpha_{0}\right|=$ 20 , which corresponds to a photon number of 400 , and investigate its quantum features. Again, we take the vacuum state as an initial state. The results for the time evolution of (a)

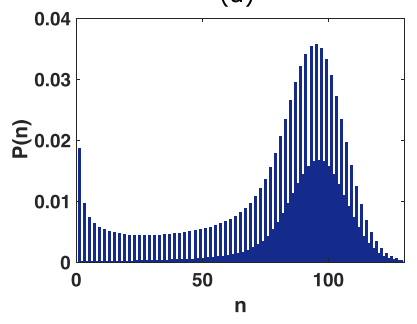

(c)
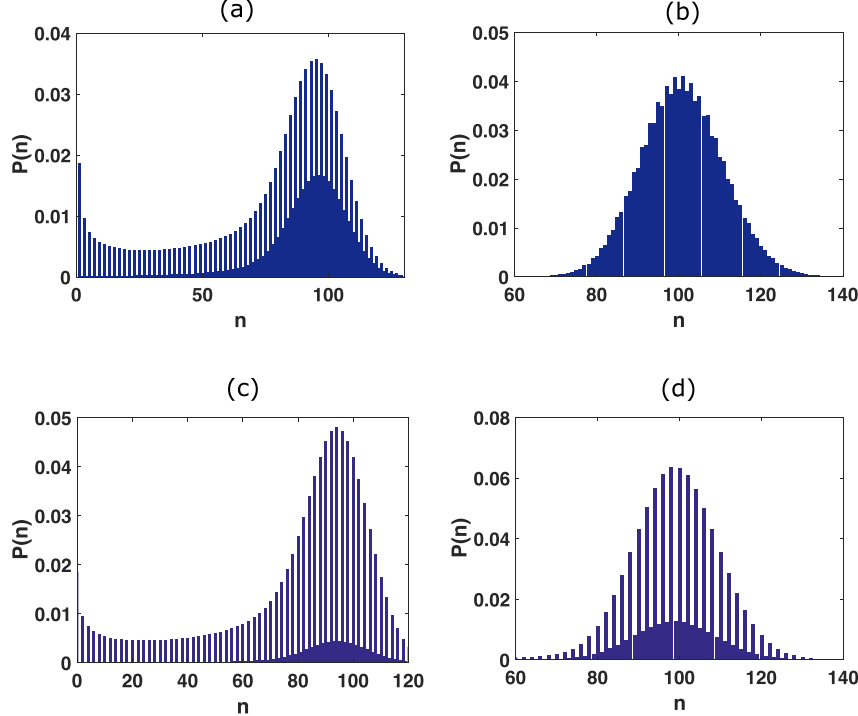

(d)

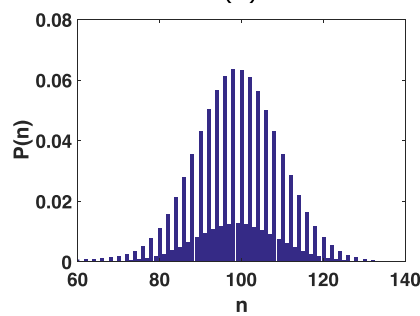

FIG. 2. The effect of squeezed reservoirs on the cat-state formation as shown by the photon number distribution. Plots show the photon number probability distribution at times $\tau=0.0075$ [(a) and (c)] and the later time $\tau=0.015[(\mathrm{~b})$ and (d)]. The parameters are $g=2.5, \lambda / g^{2}=100, N_{\text {th }}=0$, and $M=\sqrt{N_{s}\left(N_{s}+1\right)}$. (a), (b) (top) correspond to a reservoir with no squeezing. (c), (d) (lower) correspond to a squeezed reservoir $\left(N_{s}=1, M=\sqrt{2}\right)$, where we see that the probability for an odd photon number is highly suppressed.

the $p$-quadrature probability distributions for a large amplitude $\alpha_{0}=20$, for zero temperature and $\chi=0$, are shown in Fig. 6. We compare the results without squeezing and with significant squeezing $\left(N=2, M=\sqrt{6}, \Delta^{2} p=0.1 \Delta^{2} p_{\mathrm{vac}}\right)$. In accordance with the earlier results for a smaller coherent amplitude in Fig. 1, we see an enhancement of the interference fringes in the $p$-quadrature probability distribution when the reservoir is squeezed. Different to the case of no squeezing, the interference fringes persist to the end of our simulation at dimensionless time $\tau=0.0050$.

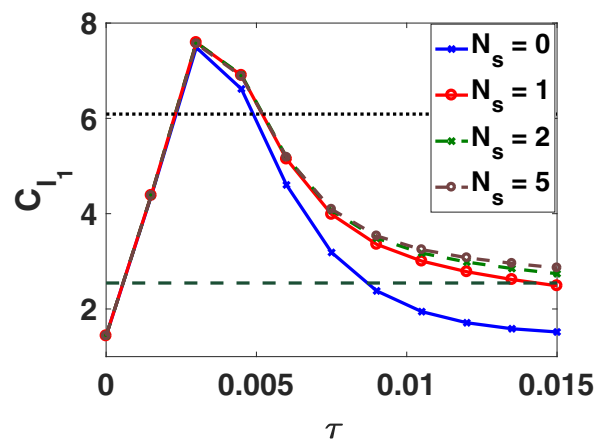

FIG. 3. The time evolution of coherence $C_{l_{1}}$ [defined in Eq. (24)] at zero temperature $\left(N_{\text {th }}=0\right)$ for different squeezing strengths. Parameters are $g=2.5, \lambda / g^{2}=100, \chi=0$, and $M=\sqrt{N_{s}\left(N_{s}+1\right)}$. The upper dashed horizontal line gives the value for a pure cat state Eq. (15) and the lower horizontal line gives the value for a mixture Eq. (16). 


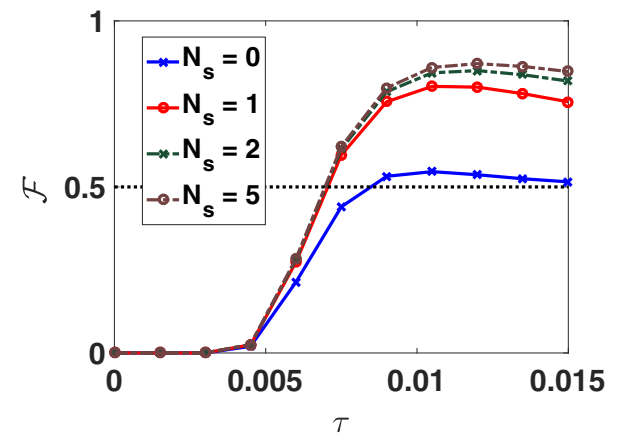

FIG. 4. The time evolution of fidelity $\mathcal{F}$ at zero temperature $\left(N_{\text {th }}=0\right)$ for different squeezing strengths. Parameters are $g=2.5$, $\lambda / g^{2}=100, \chi=0$, and $M=\sqrt{N_{s}\left(N_{s}+1\right)}$. The dotted horizontal line gives the value of $\mathcal{F}$ for a mixture Eq. (16).

\section{CAT STATES FOR MICROWAVE FIELDS WITH SQUEEZED-STATE RESERVOIRS}

We now include the thermal contribution and compare cases with and without a squeezed reservoir. While thermal noise at room temperature for optical fields is negligible, the effect of such noise is significant if present. This helps us to understand the results for the microwave regime, where thermal noise will be significant at room temperature. For

(a)

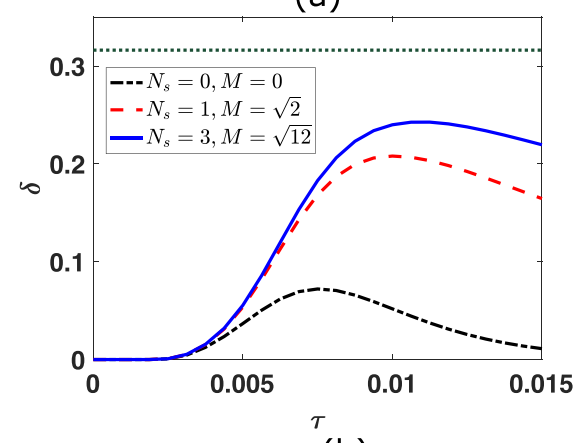

(b)

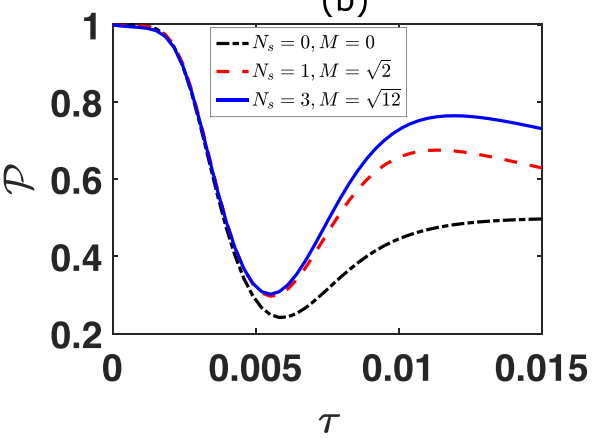

FIG. 5. The effect of squeezed reservoirs on the cat-state formation as shown by negativity and purity. The plots give the Wigner negativity $\delta$ and purity $\mathcal{P}$ as a function of the dimensionless time $\tau$, for different degrees of squeezing, at zero temperature $N_{\text {th }}=0$. $N_{s}$ is the mean photon number of the squeezed state and $M=$ $\sqrt{N_{s}\left(N_{s}+1\right)}$ determines the strength of squeezing. Parameters are $g=2.5$ and $\lambda / g^{2}=100$. The upper dashed horizontal line in the top figure gives the negativity value for a pure cat state Eq. (15). The mixture Eq. (16) has zero negativity. (a)

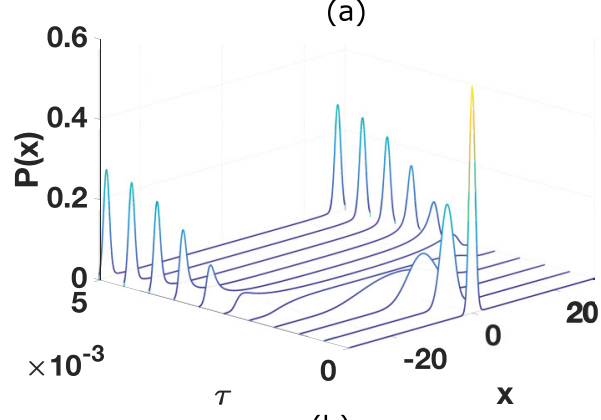

(b)

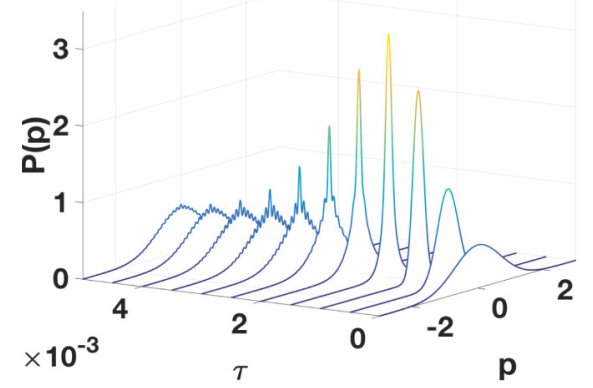

(c)

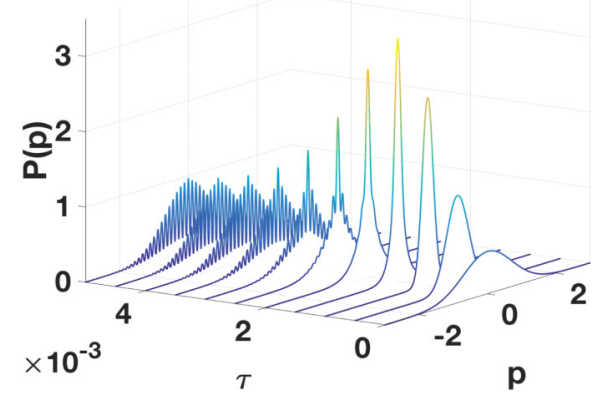

FIG. 6. Formation of a large cat state in a DPO at zero temperature with the aid of squeezing. The time evolution of the $x$ and $p$ quadratures without a squeezed reservoir are shown in plots (a) and (b). The time evolution with a squeezed reservoir $N_{s}=2, M_{s}=\sqrt{6}$ is shown (c). The plot for the $x$ quadrature is indistinguishable from plot (a), with no squeezing. The parameters are $g=2$ and $\lambda / g^{2}=$ 400 , which corresponds to a coherent amplitude of $\alpha_{0}=20$.

concreteness, we calculate the mean thermal photon number at room temperature for the signal mode using the experimental parameters of Leghtas et al. [19]. The signal mode frequency is taken to be half the pump mode frequency, which is $\omega_{s}=2 \pi \times 4.01 \mathrm{GHz}$. The mean thermal number is then given by the expression $N_{\text {th }}=1 /\left[\exp \left(\hbar \omega_{s} / k T\right)-1\right] \approx 1522$. At cryogenic temperatures of $T \sim 100 \mathrm{mK}, N_{\text {th }}$ is of course much lower, and therefore values of $N_{\text {th }}=0.05-1$ are more typical of these experiments, but thermal effects can still be very significant.

In the Appendix, we summarize results based on the solutions of Kennedy and Walls [26], analyzing the decoherence of a system initially in a cat state, which is then coupled to a thermal squeezed reservoir. We consider both types of thermal squeezing. Without squeezing, thermal noise increases it also, and the decoherence rate increases with size $\alpha$ of the cat state. With squeezing, the decoherence is slowed. However, 
for a reservoir in a thermalized squeezed state, one cannot overcome the decoherence for finite $N_{\text {th }}$ by simply increasing the amount of squeezing. A different result is obtained for a squeezed thermal input, where enhanced squeezing will eventually reduce the decoherence. This is expected since, in the latter, the thermal noise is from the preparation of the squeezed state and does not arise from the immediate environment of the cavity.

\section{A. Thermal effects on cat states}

First, we examine the case of a thermalized squeezed reservoir, which models a system where the environment is at finite temperature as the cat states evolve, but assuming the squeezed input is generated at zero temperature. Results for the quadrature probability distributions and the photon number probability distributions are shown in Figs. 7 and 8 , respectively. Even for $N_{\text {th }} \sim 0.5-1$, thermal noise has a dramatic effect in enhancing the ratio of odd to even photon numbers, thus reducing the purity. In the presence of thermal noise, the squeezing parameters that allow significant

(a)

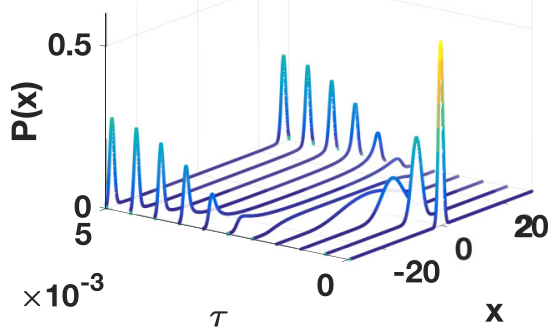

(b)

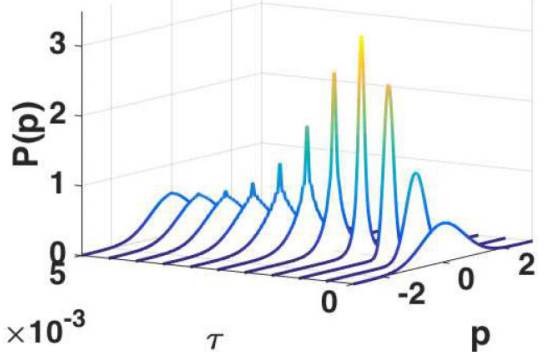

(c)

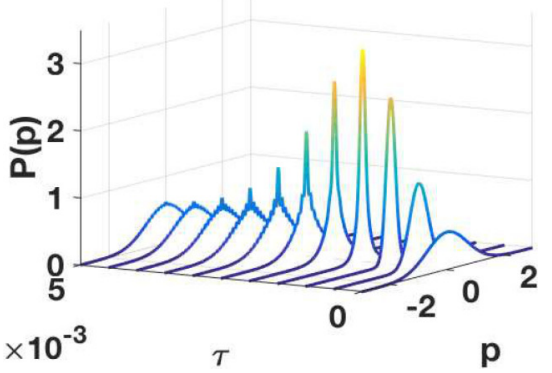

FIG. 7. Effect of thermal noise. The time evolution of the $x$ quadrature without squeezing $\left(N_{s}=M=0\right)$ is given by the top graph (a), and is unchanged for squeezing given by $N_{s}=2, M=\sqrt{6}$. The middle graph (b) shows the $p$ quadrature without squeezing, and the lower graph (c) shows the $p$ quadrature with squeezing $N_{s}=2$, $M=\sqrt{6}$. Here $N_{\text {th }}=0.5$. The parameters are as for Fig. 6 .
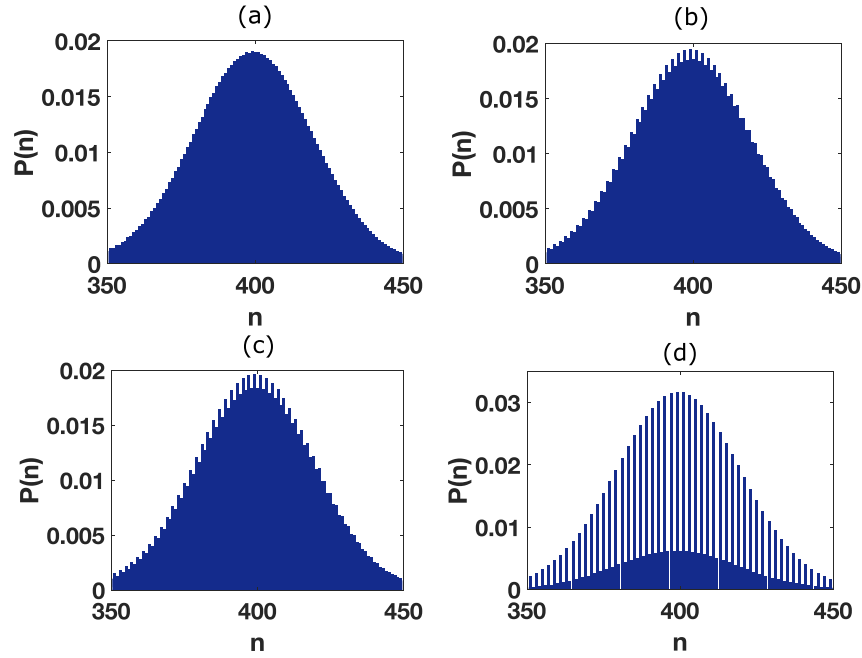

FIG. 8. Effect of thermal noise. The photon number probability distribution at $\tau=0.0050$ for the cases (a) without squeezing $(N=$ $2, M=0)$ and (b) with squeezing $\left(N_{s}=2, M=\sqrt{6}\right)$ at a finite temperature, $N_{\mathrm{th}}=0.5$. The non-Poissonian character is significantly reduced compared to the case with zero temperature $N_{\mathrm{th}}=0$, plotted directly below, for the same squeezing parameters $N_{s}=0$ (c) and $N_{s}=2, M=\sqrt{6}(\mathrm{~d})$.

improvement of the fringe visibility at zero temperature are no longer sufficient to suppress the loss of quantum coherence. The plots in Fig. 9 indicate that the effect of thermal noise is not overcome by simply introducing a large amount of squeezing, as consistent with the results given in the Appendix. This observation is also confirmed by the fidelity plots in Fig. 10. More optimistic results would be expected for a squeezed thermal input.

\section{B. Effects of Kerr nonlinearities}

Next, we extend the study to a superconducting circuit experiment where Kerr nonlinearity, represented by $\bar{\chi}$ in Eq. (1), is present. Typically, thermal noise is not negligible. As shown in previous work [57-59], the Kerr nonlinearity rotates the

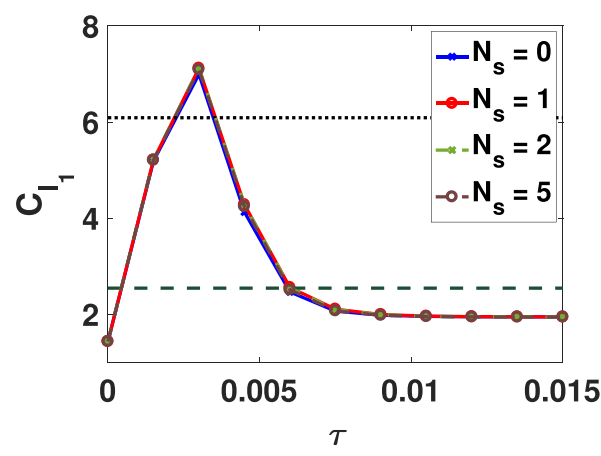

FIG. 9. The time evolution of coherence $C_{l_{1}}$ [defined in Eq. (24)] at a finite temperature corresponding to $N_{\text {th }}=1$, for different squeezing strengths. Parameters are $g=2.5, \lambda / g^{2}=100, \chi=5$, and $M=$ $\sqrt{N_{s}\left(N_{s}+1\right)}$, as in Fig. 13. The upper dashed horizontal line gives the value for a pure cat state Eq. (15) and the lower horizontal line gives the value for a mixture Eq. (16). 


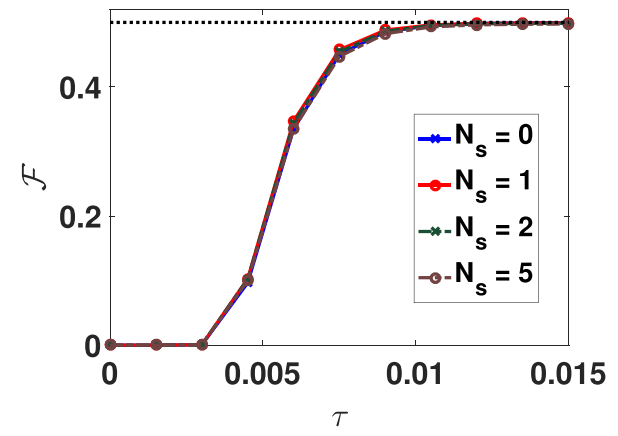

FIG. 10. The time evolution of fidelity $\mathcal{F}$ at a finite temperature corresponding to $N_{\text {th }}=1$, for different squeezing strengths. Parameters are $g=2.5, \lambda / g^{2}=100, \chi=5$, and $M=\sqrt{N_{s}\left(N_{s}+1\right)}$, as in Fig. 13. The dotted horizontal line gives the value of $\mathcal{F}$ for a mixture Eq. (16).

steady state about the origin in phase space. We will see that thermal noise induces decoherence and shortens the cat-state lifetime.

In the presence of Kerr nonlinearity, the steady state of the system in the limit of zero signal damping and for an initial vacuum state corresponds to a cat state with complex amplitude $\alpha_{0}= \pm \sqrt{\lambda /\left(g^{2}+i \chi^{\prime}\right)}$ [58]. The interference fringes in this case are observed along the $p_{\theta}$ quadrature, where $\theta$ is the phase angle of the complex number $\alpha_{0}=\left|\alpha_{0}\right| \mathrm{e}^{i \theta}$ with corresponding quadratures $x^{\prime}=x_{\theta}, p^{\prime}=p_{\theta}$. It is therefore desirable to have the reservoir in a squeezed state along the direction of the $P_{\theta}$ quadrature. This can be achieved by choosing $M=|M| \mathrm{e}^{2 i \theta}$, as can be seen in Eq. (9), with $|M|=$ $\sqrt{N_{s}\left(N_{s}+1\right)}$ giving the optimal squeezing strength.

To study the effect of the squeezed reservoir, we first consider the zero-temperature case. In Fig. 11, we observe two Gaussian peaks along the direction of the $x^{\prime}$ quadrature, indicating the formation of two coherent amplitudes with opposite phases. As previously mentioned, the quantum nature of the state is inferred from the $p^{\prime}$-quadrature probability distribution. Figure 11 shows interference fringes. The visibility of these fringes is higher with squeezing of $N_{s}=2$. This is confirmed by the time evolution of the Wigner negativity as presented in Fig. 12. We see that the Wigner negativity is larger for $N_{s}=2$ as compared to $N_{s}=0$, after the two peaks have formed along the $x^{\prime}$ quadrature around $\tau=0.002$. The corresponding purity is also higher with squeezing.

However, the same figure shows a lower Wigner negativity and purity for a larger squeezing strength $N_{s}=5$, compared to that with $N_{s}=2$. These results can be understood by realizing that, throughout the simulation, the reservoir squeezing is along the $p^{\prime}$ quadrature and antisqueezed along the $x^{\prime}$ quadrature, while the steady state of the system only reaches two coherent amplitudes with opposite phases along the $x^{\prime}$ quadrature after a certain time. In other words, before the system reaches the steady state, the squeezed reservoir adds noise with a nonoptimal orientation. This is evident from the slightly counterintuitive result of lower Wigner negativities for larger squeezing amplitudes, as shown in Fig. 12, before the development of the two probability distribution peaks along the $x^{\prime}$ quadrature. In the case of finite temperature, the thermal bath contributes further extra noise to the system. This (a)

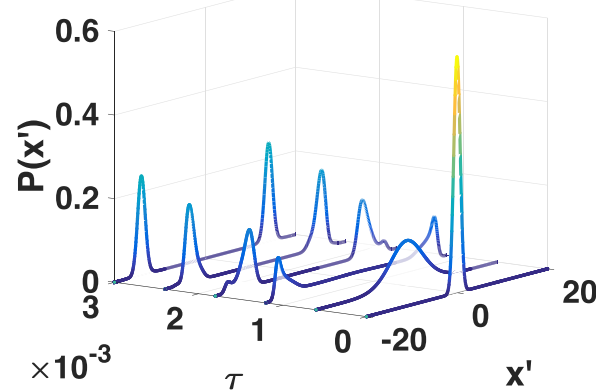

(b)

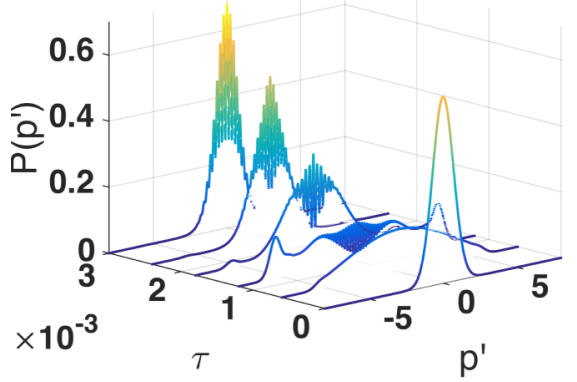

(c)

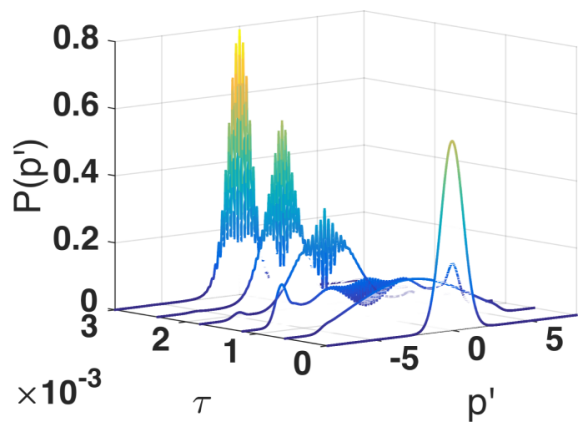

FIG. 11. Formation of cat states using squeezed reservoirs for the microwave system. The $x^{\prime}$-quadrature probability distribution and $p^{\prime}$-quadrature probability distribution are plotted as a function of dimensionless time $\tau$, assuming zero temperature $N_{\text {th }}=0$ and with Kerr nonlinearity $\chi=5$. The parameters are $g=2.5, \lambda / g^{2}=100$. The amount of squeezing is determined by $M=\sqrt{N_{s}\left(N_{s}+1\right)}$. Plot (b) is for $N_{s}=0$ (no squeezing). Plot (c) is for $N_{s}=2, M=\sqrt{6}$ (squeezing is present). The $x^{\prime}$-quadrature graphs (a) are indistinguishable for each case.

is shown in Fig. 13, where the application of the squeezed state with fixed direction has little effect on the negativity.

To overcome this effect, we can take two approaches. First, we allow the system to evolve without squeezing of reservoirs until near the time for the two distinct peaks in $x^{\prime}$ to form. For the parameters of Fig. 12, this corresponds to $\tau \sim 0.002$. At that time, we include a squeezed reservoir, the direction of squeezing being in the $p^{\prime}$ direction. This models the insertion of a squeezed state into the input port of a single-ended cavity. The results of the simulation are shown in Fig. 14, where it is seen that the squeezing gives an increased value of the negativity after that time. The second approach is to apply a squeezed state that has a varying direction, to match at a given time the direction $p^{\prime}$ orthogonal to the rotating amplitudes $\alpha_{0}$. 
(a)

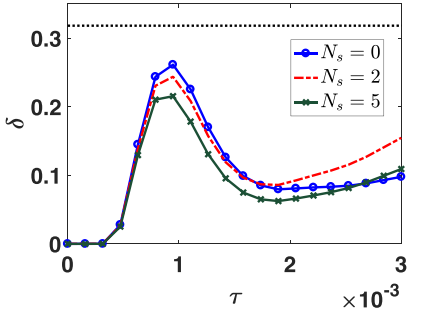

(b)

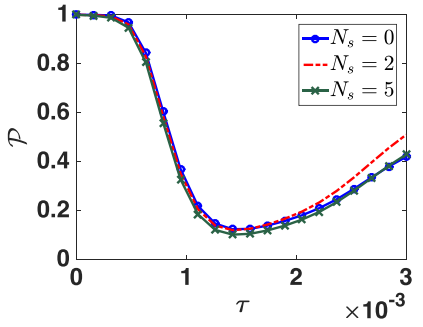

FIG. 12. The time evolution of the Wigner negativity (left) and purity (right) at zero temperature $\left(N_{\text {th }}=0\right)$ and for $\chi=5$, for different squeezing strengths. Here, the parameters are $g=2.5, \lambda / g^{2}=$ 100 and $M=\sqrt{N_{s}\left(N_{s}+1\right)}$. The negativity value corresponding to the pure cat state Eq. (15) is $\delta=0.318$ as shown in Fig. 5. The mixture Eq. (16) has zero negativity.

\section{Squeezing effects with typical experimental parameters}

Next, we look at the effects of a squeezed reservoir using experimental parameters for the superconducting circuit experiment of Leghtas et al. [19]. Here we evaluate $g=1.41$ and $\chi^{\prime}=1.01$, giving an estimated $\chi=\chi^{\prime} / g^{2}=0.5$ and $\left|\alpha_{0}\right|=2$. The mean thermal photon number is very low, at $N_{\text {th }} \leqslant 0.05 \ll 1$, which is set here at $N_{\text {th }}=0.02$. We present the $x^{\prime}$-quadrature probability distributions in Fig. 15 and observe that the two peaks along $x^{\prime}$ do not become that well separated. The simulation introduces the squeezed reservoir after $\tau=0.1$, when the two peaks are starting to develop.

The effect of adding a squeezed reservoir, which was not present in the original experiment, can be observed in Fig. 15, where we show the time evolution of the $p^{\prime}$-quadrature probability distribution. Without the squeezed reservoir, the $p^{\prime}$-quadrature probability distribution is Gaussian-like throughout the simulation. The probability distribution with squeezing displays some features of interference fringes, suggesting a quantum state with larger nonclassicality in the presence of the squeezed reservoir. This is confirmed in Fig. 16, where we compare the Wigner negativity and purity for the case without and with squeezing. With squeezing, the Wigner negativity has a larger value. Similarly, there is an increase in the purity of the quantum state.

To probe the properties of the catlike state that might be generated in a potential experiment, we next calculate the quantum coherence $C_{l_{1}}(\rho)$ for the transient catlike states ver-

(a)

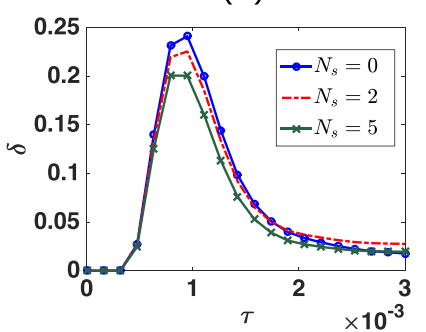

(b)

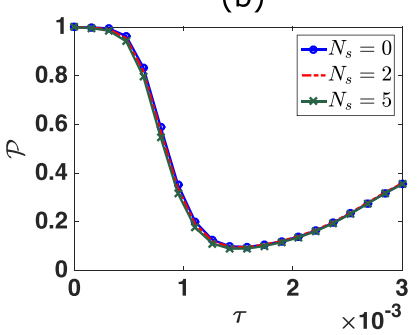

FIG. 13. The time evolution of the Wigner negativity (left) and purity (right) at a finite temperature corresponding to $N_{\text {th }}=1$, for different squeezing strengths. Parameters are $g=2.5, \lambda / g^{2}=100$, $\chi=5$, and $M=\sqrt{N_{s}\left(N_{s}+1\right)}$.

(a)

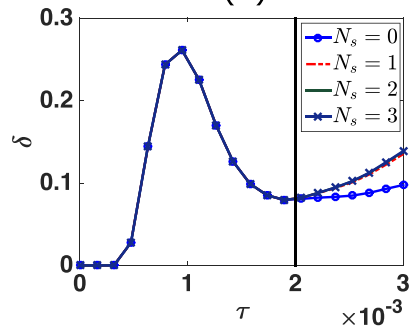

(b)

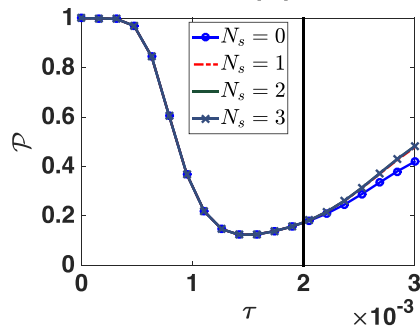

FIG. 14. The time evolution of the Wigner negativity (left) and purity (right) at zero temperature, for different squeezing strengths. Here, different from Fig. 12, the squeezed reservoir only interacts with the system at the time of formation of the two distinct peaks along the $x^{\prime}$ quadrature. The vertical line at $\tau=0.002$ is the time when the squeezed state is applied.

(a)

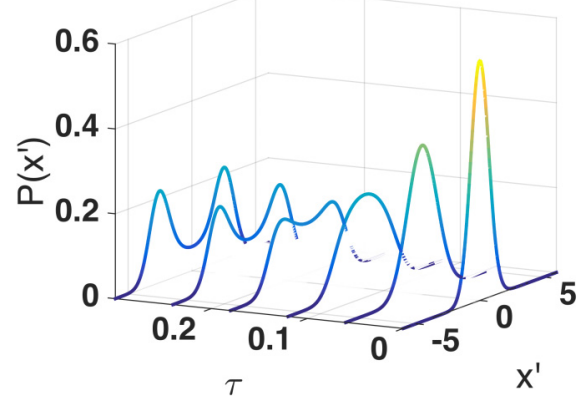

(b)

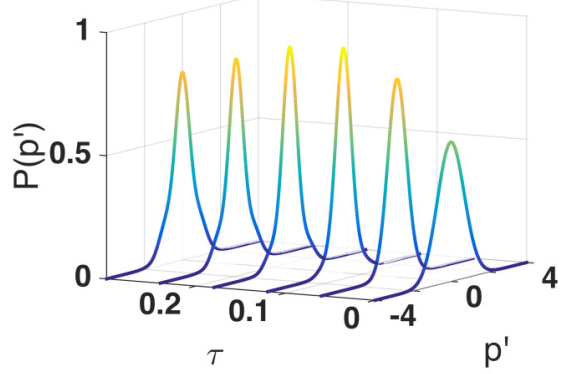

(c)

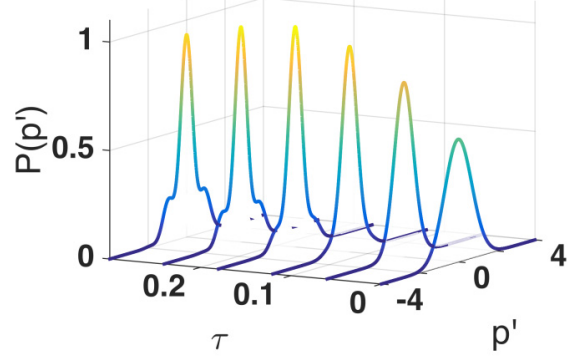

FIG. 15. The $x^{\prime}$ quadrature (a) and $p^{\prime}$ quadrature [(b), (c)] probability distributions as a function of dimensionless time $\tau$, at a finite temperature $N_{\text {th }}=0.02$. Here, the coherent amplitude is $\left|\alpha_{0}\right|=2$. The parameters are $g=1.41$ and $\chi^{\prime}=1.01$, which are taken from the experiment of Leghtas et al. [19]. Plot (b) is without squeezing, $N_{s}=0$. Plot (c) is with squeezing $N_{s}=0.5$. The $x^{\prime}$-quadrature probability distributions with and without squeezing are indistinguishable. 
(a)
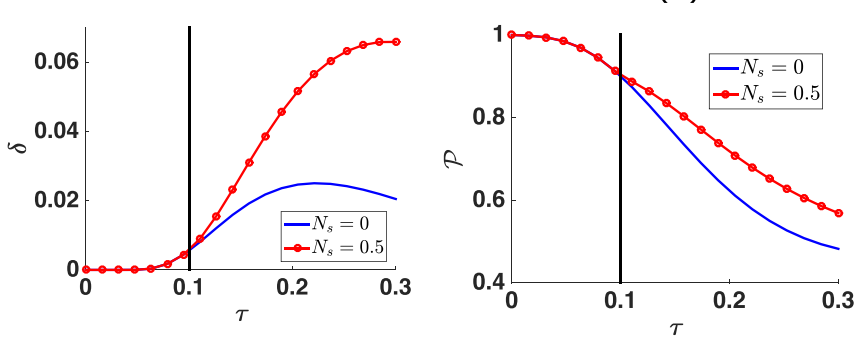

FIG. 16. The time evolution of (left) the Wigner negativity and (right) purity. The parameters are identical to those given in Fig. 15.

sus time. For the purpose of comparison, Fig. 9 gives the values of $C_{l_{1}}(\rho)$ for the pure cat state and for the mixture $\rho_{\text {mix }}$ of the two coherent states. As explained in Sec. III, the quantum coherence for the mixture arises from the quantum coherence of the coherent states $| \pm \alpha\rangle$ involved in the mixture, whereas the quantum coherence for the cat state contains the extra contribution due to the state being a macroscopic superposition. Figure 17 gives the $C_{l_{1}}(\rho)$ for the parameters of the Leghtas et al. experiment [19]. Here, the quantum coherence is at $50 \%$ of the value for an ideal cat state, significantly above that of the mixture, and it can be seen that the squeezing enhances the lifetime for a higher quantum coherence. When squeezing is present, the $C_{l_{1}}$ values are higher, implying a higher degree of quantum coherence. However, consistent with the observation that the threshold features for the cat state cannot be significantly altered by squeezing, we note that the effect of squeezing saturates, in this case at $N_{s} \sim 0.5$. Once again, these observations are consistent with the corresponding fidelity results shown in Fig. 18. Using the experimental parameters of Leghtas et al. [19], where the signal mode frequency is $\omega_{s}=2 \pi \times 4.01 \mathrm{GHz}$, and the expression $N_{\text {th }}=1 /\left[\exp \left(\hbar \omega_{s} / k T\right)-1\right]$, a mean thermal photon number $N_{\text {th }}$ of 0.02 corresponds to a temperature $T \approx 50 \mathrm{mK}$. (a)

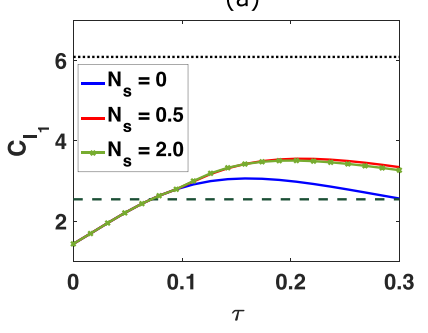

(b)

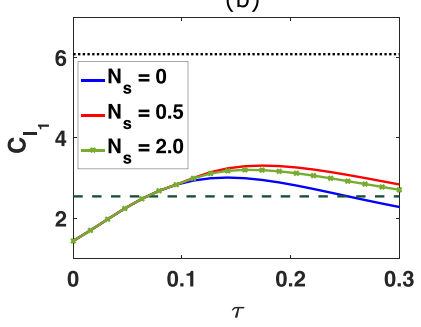

FIG. 17. The time evolution of coherence $C_{l_{1}}$ [defined in Eq. (24)] for the evolution of a catlike state using parameters similar to the experiment of Ref. [19]. For each plot, we show results with squeezing (upper red solid line) and without squeezing (lower blue solid line). The results for parameters similar to the experiment $\left(g=1.41, N_{\text {th }}=0.02, \chi^{\prime}=1.01\right)$ are shown in the figure on the right. Parameters for the left figure are $g=1.41, N_{\text {th }}=0$, $\chi^{\prime}=0$, and are given for comparison. In both figures, $\left|\alpha_{0}\right|=2$ and $M=\sqrt{N_{s}\left(N_{s}+1\right)}$ when $N_{s} \neq 0$. The upper horizontal dashed line gives the $C_{l_{1}}$ value of the corresponding cat state. The lower dashed horizontal line gives the $C_{l_{1}}$ value for mixture of coherent states with amplitude $\left|\alpha_{0}\right|=2$. (a)
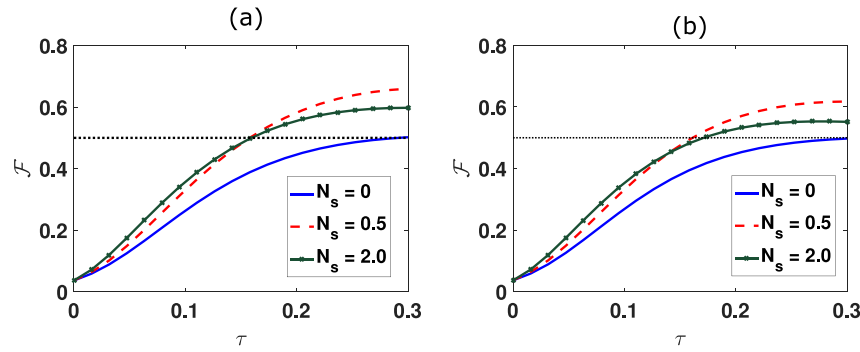

FIG. 18. The time evolution of fidelity $\mathcal{F}$ for the evolution of a catlike state using parameters similar to the experiment of Ref. [19]. For each plot, we show results with squeezing (upper red dashed line) and without squeezing (lower blue solid line). The results for parameters similar to the experiment $\left(g=1.41, N_{\text {th }}=0.02, \chi^{\prime}=1.01\right)$ are shown in the figure on the right. Parameters for the left figure are $g=1.41, N_{\text {th }}=0, \chi^{\prime}=0$, and are given for comparison. In both figures, $\left|\alpha_{0}\right|=2$ and $M=\sqrt{N_{s}\left(N_{s}+1\right)}$ when $N_{s} \neq 0$. The dotted horizontal line gives the $\mathcal{F}$ value for mixture of coherent states with amplitude $\left|\alpha_{0}\right|=2$.

\section{CONCLUSION}

In this paper, we have demonstrated the feasibility of using quantum squeezed states to enhance the macroscopic quantum coherence of dynamical cat states prepared in a cavity through a nonlinear parametric interaction. Here, we have focused on a DPO in the limit of an adiabatically eliminated pump mode, where a two-photon dissipative mechanism dominates. The cat state, which is a superposition of two coherent states with a $\pi$ phase difference, is predicted to be created in the cavity signal mode from an initial vacuum state, in the limit where there are no losses in the cavity mode. A cat state is also predicted in the microwave regime, where extra nonlinear terms are present. The loss of photons from the cavity decoheres the cat state. Thus, whether the cat state is formed or not depends on the dynamical interplay of the quantum nonlinear effect versus the signal decoherence.

We have found that it is possible to significantly limit the effects of decoherence by squeezing the input vacuum noise that enters the cavity port at the signal frequency. This amounts to controlling the fluctuations of the reservoir. The cat state then has a longer lifetime, which enables the formation of cat states of a higher purity for the same experimental parameters. Our result is consistent with earlier work on the decoherence of a pure cat state, and extends previous treatments of the effect of squeezing on cat states formed in the DPO system by giving a broader range of parameters and implementing different signatures, such as negativity and measures of quantum coherence.

We obtain results for the microwave system, which is relevant to current experiments and based on a different Hamiltonian. We find that the direction of squeezing is important. The enhancement occurs when the quantum noise of the signal cavity input is squeezed in the direction orthogonal to the axis connecting the amplitudes of the two coherent states. This rotates with the dynamics, and hence the squeezing direction needs to be controlled. Ultimately, the direction is determined by the type of nonlinearity, and whether an additional Kerr effect is present. 
Thermal noise also induces a decoherence of the cat state and has a profound effect on the feasibility of creating the cat states in the dynamical system. In the microwave regime, cooling is necessary to observe the formation of cat states. We have shown how the presence of squeezing enhances the quality of the cat state that can be formed for systems at a temperature corresponding to the microwave experiment of Leghtas et al. [19]. Our results are based on statistics modeled on a squeezed thermalized reservoir, and are consistent with earlier treatments [26,29], showing that while squeezing can inhibit the thermal decoherence, the thermal decoherence cannot be completely overcome by further increasing squeezing. This contrasts with the result in the absence of thermal noise. On the whole, based on analytical calculations where one analyses the decoherence of an ideal cat state coupled to a reservoir, we anticipate a more promising situation for a squeezed thermal state input. This models a squeezed state generated from a source at nonzero temperature, but requires that the cavity be kept at zero temperature over the timescales during which the cat state forms in the cavity.

\section{ACKNOWLEDGMENTS}

This work was performed on the OzSTAR national facility at Swinburne University of Technology. OzSTAR is funded by Swinburne University of Technology and the National Collaborative Research Infrastructure Strategy (NCRIS). P.D.D. and M.D.R. thank the hospitality of the Weizmann Institute of Science. This work was funded through Australian Research Council Discovery Project Grants No. DP180102470 and No. DP190101480. The authors wish to thank NTT Research for their financial and technical support.

\section{APPENDIX}

\section{Statistical moments for a squeezed thermal state}

For simplicity, here we consider the case of a representative input mode $b$ near resonance rather than the full multimode squeezed reservoir. The general input squeezed state is more complex than this, as it is nondegenerate [41], with correlated input modes $b_{0}(\omega)$ paired around $\omega=\omega_{0} \pm \Delta \omega$.

A squeezed thermal state has a corresponding density operator $[61,62]$

$$
\begin{aligned}
\rho_{S T} & =S \rho_{T} S^{\dagger} \\
& =\frac{1}{1+N_{\mathrm{th}}} \sum_{n}\left(\frac{N_{\mathrm{th}}}{1+N_{\mathrm{th}}}\right)^{n} S|n\rangle\langle n| S^{\dagger},
\end{aligned}
$$

where $S(\epsilon)=\exp \left[\left(\epsilon^{*} b^{2}-\epsilon b^{\dagger 2}\right) / 2\right]$ is the squeezing operator, $\epsilon=r e^{i \phi}$, and $N_{\text {th }}$ is the mean photon number of a thermal state. The mean photon number for the squeezed thermal state is

$$
\left\langle b^{\dagger} b\right\rangle=N_{\text {th }} \cosh 2 r+\sinh ^{2} r,
$$

while other statistical moments are given by

$$
\left\langle b^{2}\right\rangle=-\frac{1}{2} \sinh 2 r e^{i \phi}\left(2 N_{\mathrm{th}}+1\right)
$$

and

$$
\left\langle b^{\dagger 2}\right\rangle=-\frac{1}{2} \sinh 2 r e^{-i \phi}\left(2 N_{\text {th }}+1\right) .
$$

Comparing these equations with the statistical moments for the environment mode operators in Eqs. (6), we see that, for a squeezed thermal state,

$$
\begin{aligned}
N & =N_{\mathrm{th}} \cosh 2 r+\sinh ^{2} r, \\
M & =-\frac{1}{2} \sinh 2 r e^{i \phi}\left(2 N_{\mathrm{th}}+1\right) \\
& =\frac{1}{2}\left(2 N_{\mathrm{th}}+1\right) \sinh 2 r e^{i(\phi-\pi)} .
\end{aligned}
$$

In particular, for $\theta=0$, the variances of $X$ and $P$ quadratures as defined in Eqs. (7) have the expressions

$$
\begin{aligned}
\Delta^{2} X= & \left(N_{\text {th }} \cosh 2 r+\sinh ^{2} r+\frac{1}{2}\right) \\
& +\frac{1}{2} \sinh 2 r\left(2 N_{\text {th }}+1\right) \cos (\phi-\pi), \\
\Delta^{2} P= & \left(N_{\text {th }} \cosh 2 r+\sinh ^{2} r+\frac{1}{2}\right) \\
& -\frac{1}{2} \sinh 2 r\left(2 N_{\text {th }}+1\right) \cos (\phi-\pi) .
\end{aligned}
$$

To get a squeezing in the $P$ quadrature, we set $\phi=\pi[M$ is real with amplitude $\left.\left(N_{\text {th }}+1 / 2\right) \sinh 2 r\right]$ and Eqs. (A1) are then

$$
\Delta^{2} X=\left(N_{\text {th }}+\frac{1}{2}\right) e^{2 r}, \quad \Delta^{2} P=\left(N_{\text {th }}+\frac{1}{2}\right) e^{-2 r} .
$$

We note that the thermal contribution is squeezed (antisqueezed) by the factor of $e^{-2 r}\left(e^{2 r}\right)$. This means that such thermal squeezing (if in the direction so as to squeeze fluctuations in $P$ ) would give significant enhancement of the observed macroscopic quantum coherence. We do not give explicit calculation of such enhancement in this paper, but the calculations could be done in principle by substituting for $N$ and $M$.

\section{Statistical moments for a thermalized squeezed state}

As given above, we only consider a representative squeezed mode $b$ near resonance. Fearn and Collett [61] defined a thermalized squeezed state with a density operator in the coherent basis (Glauber-P representation) as follows:

$$
\rho_{T S}=\frac{1}{\pi N_{\mathrm{th}}} \int d^{2} \beta \exp \left(-\frac{|\beta|^{2}}{N_{\mathrm{th}}}\right)|\beta, \epsilon\rangle\langle\beta, \epsilon|,
$$

where $|\beta, \epsilon\rangle=D(\beta) S(\epsilon)|0\rangle=S(\epsilon) D(\gamma)|0\rangle$ is a displaced squeezed state, $D(\beta)$ is the displacement operator, $S(\epsilon)$ is the squeezing operator as defined in the previous section, and $\gamma=\beta \cosh r+\beta^{*} e^{i \phi} \sinh r$.

The mean photon number for the thermalized squeezed state is

$$
\left\langle b^{\dagger} b\right\rangle=\sinh ^{2} r+N_{\mathrm{th}}
$$

and the statistical moments $\left\langle b^{2}\right\rangle$ and $\left\langle b^{\dagger 2}\right\rangle$ are

$$
\left\langle b^{2}\right\rangle=\frac{1}{2} \sinh 2 r e^{i(\phi-\pi)}, \quad\left\langle b^{\dagger 2}\right\rangle=\frac{1}{2} \sinh 2 r e^{-i(\phi-\pi)} .
$$

Comparing these equations with the statistical moments for the environment mode operators in Eqs. (6), we see that, for a thermalized squeezed state:

$$
N=N_{\text {th }}+\sinh ^{2} r, \quad M=\frac{1}{2} \sinh 2 r e^{i(\phi-\pi)} .
$$

For $\theta=0$ and $\phi=\pi$, the variances of $X$ and $P$ quadratures as defined in Eqs. (7) have the expressions

$$
\Delta^{2} X=2 N_{\text {th }}+e^{2 r}, \quad \Delta^{2} P=2 N_{\text {th }}+e^{-2 r} .
$$




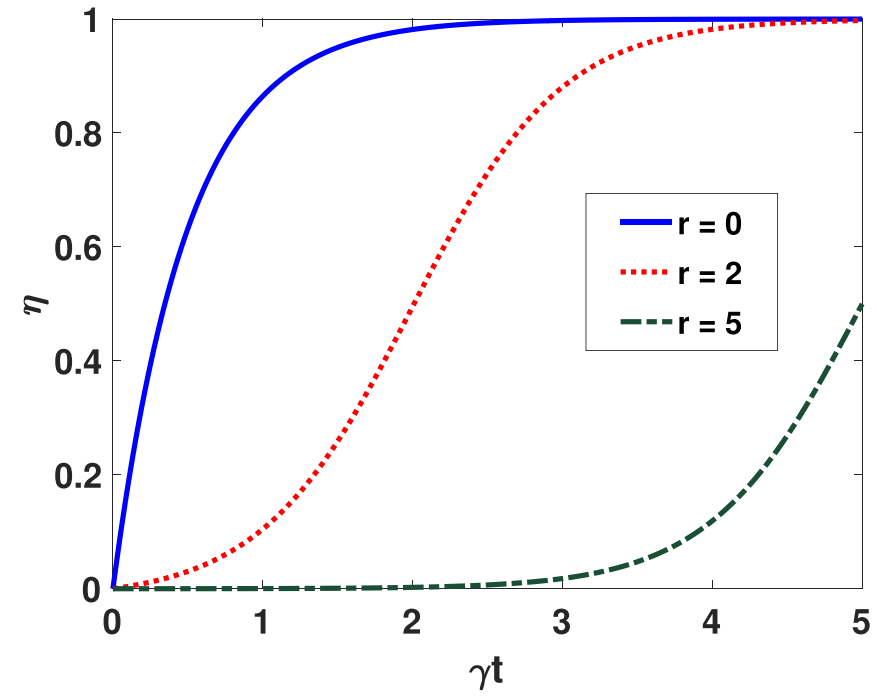

FIG. 19. The term that contributes to the interference pattern $\eta$ as a function of $\gamma t . \eta=0$ demonstrates full visibility of the interference fringes. The visibility is zero when $\eta=1$. This figure corresponds to a system with zero temperature. Squeezing increases with $r$, the squeeze parameter.

As opposed to the squeezed thermal state, the thermal number $N_{\text {th }}$ in Eq. (A4) is not affected by the squeezing operation, implying that the squeezing is weaker for a thermalized squeezed state with the same squeezing strength $r$ as in the squeezed thermal state.

\section{Effect of thermal noise on decoherence of a system prepared initially in a cat state}

Kennedy and Walls [26] investigated the effect of squeezing on the probability distribution interference visibility. They considered an initial cat state with a density operator $\rho(0)=$ $\mathcal{N}\left(e^{-i \pi / 4}|\alpha\rangle+e^{i \pi / 4}|-\alpha\rangle\right)\left(e^{i \pi / 4}\langle\alpha|+e^{-i \pi / 4}\langle-\alpha|\right)$. The term in the probability distribution for $x_{\theta}$ that contributes to the interference fringes is found to be proportional to $|\langle\alpha \mid-\alpha\rangle|^{\eta}$ :

$$
\left\langle x_{\theta}|\rho(t)| x_{\theta}\right\rangle \propto|\langle\alpha \mid-\alpha\rangle|^{\eta}=\exp \left(-2|\alpha|^{\eta}\right),
$$

where

$$
\eta=1-\frac{e^{-2 \gamma t}}{\left\{1+2[N+|M| \cos (2 \theta+\Phi)]\left(1-e^{-2 \gamma t}\right)\right\}} .
$$

Here, $\theta$ determines the phase quadrature $x_{\theta}, \Phi$ is the phase of the complex parameter $M$, as previously defined, and $N=$ $N_{\text {th }}+N_{s}$. For the cat state considered, it is optimal to choose $\cos (2 \theta+\phi)=-1$.

We now summarize the results for the two types of thermal states considered above. We examine results for the type of squeezing that comes from a DPO, denoting the squeezing parameter by $r$, where $M=\sqrt{N_{s}\left(N_{s}+1\right)}$. For a thermalized squeezed bath, $N=N_{\text {th }}+\sinh ^{2} r=N_{\text {th }}+N_{s}, r$ is the squeezing strength, and $|M|=\frac{1}{2} \sinh 2 r$ as calculated in the above. For a squeezed thermal bath, $N=N_{\text {th }} \cosh 2 r+\sinh ^{2} r$ and $|M|=\frac{1}{2}\left(2 N_{\text {th }}+1\right) \sinh 2 r$. Figure 19 shows the decoherence where there is damping but no thermal noise, so $N_{\text {th }}=$ 0 . In this limit, the decoherence can be totally suppressed

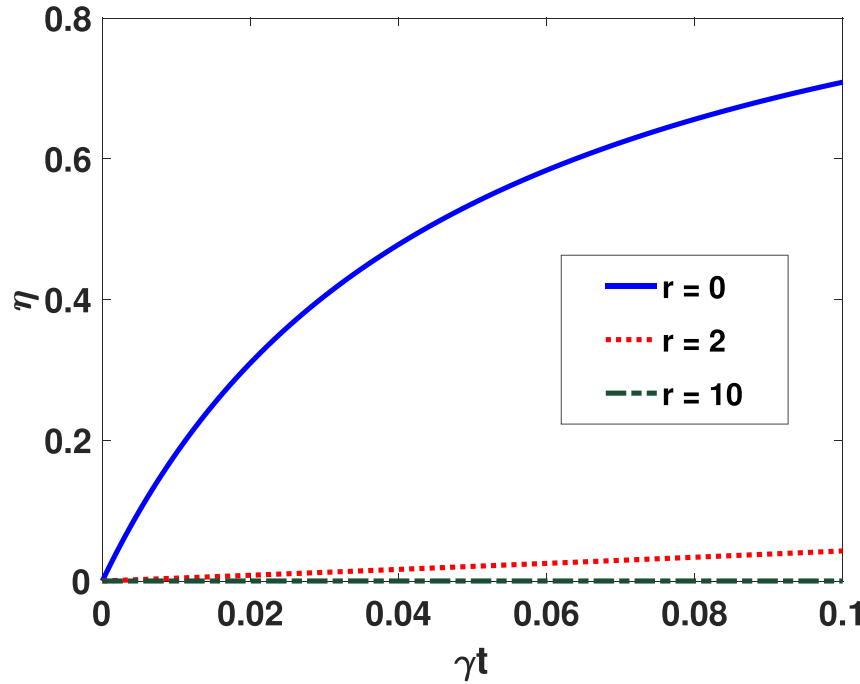

FIG. 20. Here, $\eta=0$ demonstrates full visibility of interference fringes. The visibility is zero when $\eta=1$. This figure corresponds to a squeezed thermal state at a finite temperature with $N_{\text {th }}=5$.

for longer times for sufficiently strong squeezing, where $N_{s} \rightarrow \infty$.

The results for a squeezed thermal state are given in Fig. 20. Here, we see on comparing Eqs. (A2) with (A4) that the effect of the thermal noise term $N_{\text {th }}$ for the squeezed thermal state is itself reduced by increasing the squeezing parameter. This arises because the technique of preparation is to reduce, or squeeze, the thermal noise at the squeezing source. From the figure, we see that with a sufficient amount of squeezing it is possible to completely suppress the decoherence due to thermal noise for this type of squeezed thermal input.

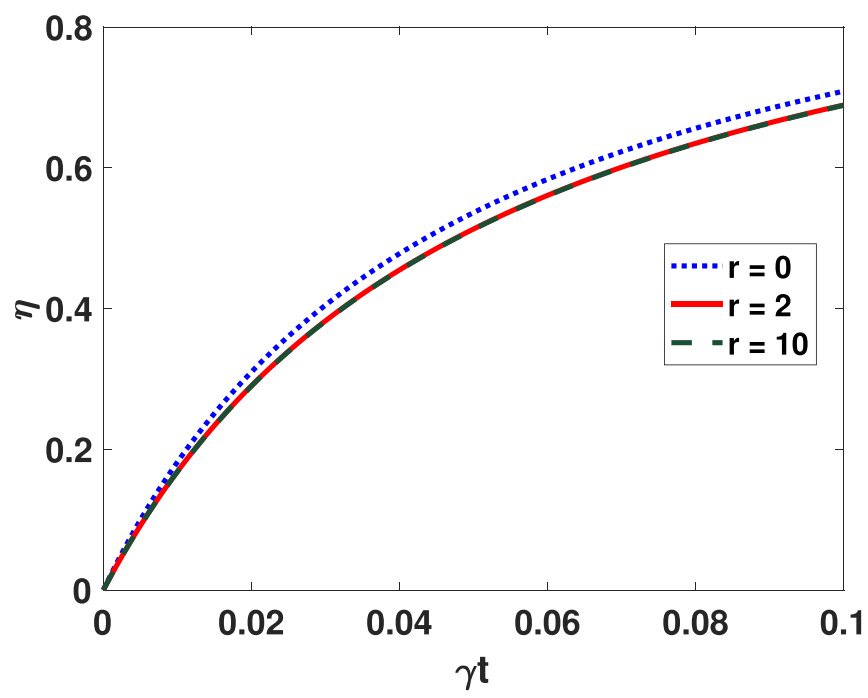

FIG. 21. Here, $\eta=0$ demonstrates full visibility of interference fringes. The visibility is zero when $\eta=1$. This figure corresponds to a thermalized squeezed state at a finite temperature with $N_{\text {th }}=5$. 
Looking at the thermalized squeezed state, we find for finite $N_{\text {th }}$ and infinite squeezing

$$
\eta \rightarrow 1-\frac{e^{-2 \gamma t}}{\left\{1+2 N_{\mathrm{th}}\left(1-e^{-2 \gamma t}\right)\right\}} .
$$

For long times, $\eta \rightarrow 1-\frac{e^{-2 \gamma t}}{\left\{1+2 N_{\mathrm{th}}\right\}}$. This shows that thermal noise (where $N_{\text {th }}$ is fixed) remains significant in increasing decoherence. For short times, we obtain a linear response of $\eta$ with $\gamma t, \eta \sim 4 N_{\text {th }} \gamma t$. These features are observed in Fig. 21. Consistent with results reported by Serafini et al. [29], we also note that increasing squeezing does not always lead to a decrease of decoherence over a certain time.

\section{Fidelity of a mixed state with respect to a pure, even cat state}

The fidelity between a pure, even cat state $\left|\psi_{c}\right\rangle=$ $\mathcal{N}\left(\left|\alpha_{0}\right\rangle+\left|-\alpha_{0}\right\rangle\right)$ in Eq. (15) and a mixed state $\rho_{\text {mix }}=$ $\left(\left|\alpha_{0}\right\rangle\left\langle\alpha_{0}|+|-\alpha_{0}\right\rangle\left\langle-\alpha_{0}\right|\right) / 2$ is

$$
\begin{aligned}
\mathcal{F}_{\text {mix }} & =\left\langle\psi_{c}\left|\rho_{\text {mix }}\right| \psi_{c}\right\rangle \\
& =\mathcal{N}^{2}\left(1+\left\langle\alpha_{0} \mid-\alpha_{0}\right\rangle+\left\langle-\alpha_{0} \mid \alpha_{0}\right\rangle+\left|\left\langle\alpha_{0} \mid-\alpha_{0}\right\rangle\right|^{2}\right) \\
& =\mathcal{N}^{2}\left(1+2\left\langle\alpha_{0} \mid-\alpha_{0}\right\rangle+\left|\left\langle\alpha_{0} \mid-\alpha_{0}\right\rangle\right|^{2}\right) \\
& =\mathcal{N}^{2}\left(1+\left\langle\alpha_{0} \mid-\alpha_{0}\right\rangle\right)^{2}=\frac{1}{2}\left(1+e^{-2\left|\alpha_{0}\right|^{2}}\right),
\end{aligned}
$$

where $\quad\left\langle\alpha_{0} \mid-\alpha_{0}\right\rangle=\left\langle-\alpha_{0} \mid \alpha_{0}\right\rangle=e^{-2\left|\alpha_{0}\right|^{2}} \quad$ and $\quad \mathcal{N}=$ $\left[2\left(1+e^{-2\left|\alpha_{0}\right|^{2}}\right)\right]^{-1 / 2}$ is the normalization constant.
[1] E. Schrödinger, Die gegenwärtige situation in der quantenmechanik (The present situation in quantum mechanics), Naturwiss. 23, 823 (1935).

[2] M. Brune, E. Hagley, J. Dreyer, X. Maître, A. Maali, C. Wunderlich, J. M. Raimond, and S. Haroche, Observing the Progressive Decoherence of the "Meter" in a Quantum Measurement, Phys. Rev. Lett. 77, 4887 (1996).

[3] C. Monroe, D. M. Meekhof, B. E. King, and D. J. Wineland, A "Schrödinger cat" superposition state of an atom, Science 272, 1131 (1996).

[4] A. Ourjoumtsev, H. Jeong, R. Tualle-Brouri, and P. Grangier, Generation of optical 'Schrödinger cats' from photon number states, Nature 448, 784 (2007).

[5] A. Palacios-Laloy, F. Mallet, F. Nguyen, P. Bertet, D. Vion, D. Esteve, and A. N. Korotkov, Experimental violation of a Bell's inequality in time with weak measurement, Nat. Phys. 6, 442 (2010).

[6] B. Vlastakis, G. Kirchmair, Z. Leghtas, S. E. Nigg, L. Frunzio, S. M. Girvin, M. Mirrahimi, M. H. Devoret, and R. J. Schoelkopf, Deterministically encoding quantum information using 100-photon Schrödinger cat states, Science 342, 607 (2013).

[7] C. Wang, Y. Y. Gao, P. Reinhold, R. W. Heeres, N. Ofek, K. Chou, C. Axline, M. Reagor, J. Blumoff, K. M. Sliwa, L. Frunzio, S. M. Girvin, L. Jiang, M. Mirrahimi, M. H. Devoret, and R. J. Schoelkopf, A Schrödinger cat living in two boxes, Science 352, 1087 (2016).

[8] A. J. Leggett and A. Garg, Quantum Mechanics Versus Macroscopic Realism: Is the Flux There When Nobody Looks? Phys. Rev. Lett. 54, 857 (1985).

[9] B. Yurke and D. Stoler, Generating Quantum Mechanical Superpositions of Macroscopically Distinguishable States via Amplitude Dispersion, Phys. Rev. Lett. 57, 13 (1986).

[10] G. C. Knee, K. Kakuyanagi, M.-C. Yeh, Y. Matsuzaki, H. Toida, H. Yamaguchi, S. Saito, A. J. Leggett, and W. J. Munro, A strict experimental test of macroscopic realism in a superconducting flux qubit, Nat. Commun. 7, 13253 (2016).

[11] A. Asadian, C. Brukner, and P. Rabl, Probing Macroscopic Realism via Ramsey Correlation Measurements, Phys. Rev. Lett. 112, 190402 (2014).

[12] C. Budroni, G. Vitagliano, G. Colangelo, R. J. Sewell, O. Gühne, G. Tóth, and M. W. Mitchell, Quantum Nondemolition
Measurement Enables Macroscopic Leggett-Garg Tests, Phys. Rev. Lett. 115, 200403 (2015).

[13] B. Opanchuk, L. Rosales-Zárate, R. Y. Teh, and M. D. Reid, Quantifying the mesoscopic quantum coherence of approximate noon states and spin-squeezed two-mode Bose-Einstein condensates, Phys. Rev. A 94, 062125 (2016); L. Rosales-Zárate, B. Opanchuk, Q. Y. He, and M. D. Reid, Leggett-Garg tests of macrorealism for bosonic systems including double-well Bose-Einstein condensates and atom interferometers, ibid. 97, 042114 (2018); M. Thenabadu and M. D. Reid, Leggett-Garg tests of macrorealism for dynamical cat states evolving in a nonlinear medium, ibid. 99, 032125 (2019).

[14] M. R. Vanner, I. Pikovski, G. D. Cole, M. Kim, Č. Brukner, K. Hammerer, G. J. Milburn, and M. Aspelmeyer, Pulsed quantum optomechanics, Proc. Natl. Acad. Sci. 108, 16182 (2011).

[15] R. Y. Teh, S. Kiesewetter, P. D. Drummond, and M. D. Reid, Creation, storage, and retrieval of an optomechanical cat state, Phys. Rev. A 98, 063814 (2018).

[16] A. Bassi, K. Lochan, S. Satin, T. P. Singh, and H. Ulbricht, Models of wave-function collapse, underlying theories, and experimental tests, Rev. Mod. Phys. 85, 471 (2013).

[17] A. O. Caldeira and A. J. Leggett, Influence of damping on quantum interference: An exactly soluble model, Phys. Rev. A 31, 1059 (1985); D. F. Walls and G. J. Milburn, Effect of dissipation on quantum coherence, ibid. 31, 2403 (1985).

[18] Z. Leghtas, G. Kirchmair, B. Vlastakis, M. H. Devoret, R. J. Schoelkopf, and M. Mirrahimi, Deterministic protocol for mapping a qubit to coherent state superpositions in a cavity, Phys. Rev. A 87, 042315 (2013).

[19] Z. Leghtas, S. Touzard, I. M. Pop, A. Kou, B. Vlastakis, A. Petrenko, K. M. Sliwa, A. Narla, S. Shankar, M. J. Hatridge, M. Reagor, L. Frunzio, R. J. Schoelkopf, M. Mirrahimi, and M. H. Devoret, Confining the state of light to a quantum manifold by engineered two-photon loss, Science 347, 853 (2015).

[20] M. Mirrahimi, Z. Leghtas, V. V. Albert, S. Touzard, R. J. Schoelkopf, L. Jiang, and M. H. Devoret, Dynamically protected cat-qubits: A new paradigm for universal quantum computation, New J. Phys. 16, 045014 (2014).

[21] M. Wolinsky and H. J. Carmichael, Quantum Noise in the Parametric Oscillator: From Squeezed States to Coherent-State Superpositions, Phys. Rev. Lett. 60, 1836 (1988). 
[22] M. D. Reid and B. Yurke, Effect of bistability and superpositions on quantum statistics in degenerate parametric oscillation, Phys. Rev. A 46, 4131 (1992).

[23] E. E. Hach III and C. C. Gerry, Generation of mixtures of Schrödinger-cat states from a competitive two-photon process, Phys. Rev. A 49, 490 (1994).

[24] L. Gilles, B. M. Garraway, and P. L. Knight, Generation of nonclassical light by dissipative two-photon processes, Phys. Rev. A 49, 2785 (1994).

[25] H. J. Carmichael, Statistical Methods in Quantum Optics 1: Master Equations and Fokker-Planck Equations (SpringerVerlag, Berlin, Heidelberg, 2013).

[26] T. A. B. Kennedy and D. F. Walls, Squeezed quantum fluctuations and macroscopic quantum coherence, Phys. Rev. A 37, 152 (1988)

[27] S. Haroche, Quantum information in cavity quantum electrodynamics: Logical gates, entanglement engineering and 'Schrödinger-cat states', Philos. Trans. R. Soc. London, Ser. A 361, 1339 (2003).

[28] F. A. A. El-Orany, J. Peřina, V. Peřinová, and M. S. Abdalla, Evolution of cat states in a dissipative parametric amplifier: Decoherence and entanglement, Eur. Phys. J. D 22, 141 (2003).

[29] A. Serafini, S. De Siena, and F. Illuminati, Decoherence of number states in phase-sensitive reservoirs, Mod. Phys. Lett. B 18, 687 (2004); A. Serafini, S. De Siena, F. Illuminati, and M. G. A. Paris, Minimum decoherence cat-like states in Gaussian noisy channels, J. Opt. B: Quantum Semiclassical Opt. 6, S591 (2004); A. Serafini, M. G. A. Paris, F. Illuminati, and S. De Siena, Quantifying decoherence in continuous variable systems, ibid. 7, R19 (2005).

[30] J. Paavola, M. J. W. Hall, M. G. A. Paris, and S. Maniscalco, Finite-time quantum-to-classical transition for a Schrödingercat state, Phys. Rev. A 84, 012121 (2011).

[31] L. Rosales-Zárate, R. Y. Teh, S. Kiesewetter, A. Brolis, K. $\mathrm{Ng}$, and M. D. Reid, Decoherence of Einstein-Podolsky-Rosen steering, JOSA B 32, A82 (2015).

[32] Q.-S. Tan, J.-Q. Liao, X. Wang, and F. Nori, Enhanced interferometry using squeezed thermal states and even or odd states, Phys. Rev. A 89, 053822 (2014).

[33] J. S. Bennett and W. P. Bowen, Rapid mechanical squeezing with pulsed optomechanics, New J. Phys. 20, 113016 (2018).

[34] H. P. Yuen, Two-photon coherent states of the radiation field, Phys. Rev. A 13, 2226 (1976).

[35] C. M. Caves, Quantum-mechanical noise in an interferometer, Phys. Rev. D 23, 1693 (1981).

[36] G. Milburn and D. Walls, Production of squeezed states in a degenerate parametric amplifier, Opt. Commun. 39, 401 (1981).

[37] B. Yurke, Use of cavities in squeezed-state generation, Phys. Rev. A 29, 408 (1984); B. Yurke and J. S. Denker, Quantum network theory, ibid. 29, 1419 (1984); M. J. Collett and C. W. Gardiner, Squeezing of intracavity and traveling-wave light fields produced in parametric amplification, ibid. 30, 1386 (1984).

[38] M. D. Reid and D. F. Walls, Quantum statistics of degenerate four wave mixing, Opt. Commun. 50, 406 (1984); M. D. Reid, D. F. Walls, and B. J. Dalton, Squeezing of Quantum Fluctuations via Atomic Coherence Effects, Phys. Rev. Lett. 55, 1288 (1985).
[39] C. Fabre, E. Giacobino, A. Heidmann, L. Lugiato, S. Reynaud, M. Vadacchino, and W. Kaige, Squeezing in detuned degenerate optical parametric oscillators, Quantum Opt.: J. Eur. Opt. Soc. B 2, 159 (1990).

[40] R. Movshovich, B. Yurke, P. G. Kaminsky, A. D. Smith, A. H Silver, R. W. Simon, and M. V. Schneider, Observation of ZeroPoint Noise Squeezing via a Josephson-Parametric Amplifier, Phys. Rev. Lett. 65, 1419 (1990).

[41] P. D. Drummond and Z. Ficek, Quantum Squeezing, Vol. 27 (Springer-Verlag, Berlin, 2004).

[42] T. P. Purdy, P.-L. Yu, R. W. Peterson, N. S. Kampel, and C. A. Regal, Strong Optomechanical Squeezing of Light, Phys. Rev. X 3, 031012 (2013).

[43] E. E. Wollman, C. U. Lei, A. J. Weinstein, J. Suh, A. Kronwald, F. Marquardt, A. A. Clerk, and K. C. Schwab, Quantum squeezing of motion in a mechanical resonator, Science 349, 952 (2015).

[44] M. A. Castellanos-Beltran, K. D. Irwin, G. C. Hilton, L. R. Vale, and K. W. Lehnert, Amplification and squeezing of quantum noise with a tunable Josephson metamaterial, Nat. Phys. 4, 929 (2008).

[45] R. E. Slusher, L. W. Hollberg, B. Yurke, J. C. Mertz, and J. F. Valley, Observation of Squeezed States Generated by fOur-Wave Mixing in an Optical Cavity, Phys. Rev. Lett. 55, 2409 (1985); A. Heidmann, R. J. Horowicz, S. Reynaud, E. Giacobino, C. Fabre, and G. Camy, Observation of Quantum Noise Reduction on Twin Laser Beams, ibid. 59, 2555 (1987); H. Vahlbruch, M. Mehmet, S. Chelkowski, B. Hage, A. Franzen, N. Lastzka, S. Goßler, K. Danzmann, and R. Schnabel, Observation of Squeezed Light with 10-db Quantum-Noise Reduction, ibid. 100, 033602 (2008); H. Vahlbruch, M. Mehmet, K. Danzmann, and R. Schnabel, Detection of $15 \mathrm{db}$ Squeezed States of Light and Their Application for the Absolute Calibration Of Photoelectric Quantum Efficiency, ibid. 117, 110801 (2016).

[46] M. Tse, H. Yu, N. Kijbunchoo, A. Fernandez-Galiana, P. Dupej, L. Barsotti, C. D. Blair, D. D. Brown, S. E. Dwyer, A. Effler et al., Quantum-Enhanced Advanced LIGO Detectors in the Era of Gravitational-Wave Astronomy, Phys. Rev. Lett. 123, 231107 (2019).

[47] M. A. Taylor, J. Janousek, V. Daria, J. Knittel, B. Hage, H.A. Bachor, and W. P. Bowen, Biological measurement beyond the quantum limit, Nat. Photonics 7, 229 (2013); F. Toscano, D. A. R. Dalvit, L. Davidovich, and W. H. Zurek, Sub-planck phase-space structures and Heisenberg-limited measurements, Phys. Rev. A 73, 023803 (2006); P. Tombesi and A. Mecozzi, Generation of macroscopically distinguishable quantum states and detection by the squeezed-vacuum technique, J. Opt. Soc. Am. B 4, 1700 (1987); A. S. Lane, M. D. Reid, and D. F. Walls, Absorption Spectroscopy Beyond the Shot-Noise Limit, Phys. Rev. Lett. 60, 1940 (1988); C. W. Gardiner, Inhibition of Atomic Phase Decays by Squeezed Light: A Direct Effect of Squeezing, ibid. 56, 1917 (1986); P. Tombesi and D. Vitali, Physical realization of an environment with squeezed quantum fluctuations via quantum-nondemolition-mediated feedback, Phys. Rev. A 50, 4253 (1994); S. L. Braunstein and H. J. Kimble, Teleportation of Continuous Quantum Variables, Phys. Rev. Lett. 80, 869 (1998). 
[48] A. Mecozzi and P. Tombesi, Distinguishable Quantum States Generated via Nonlinear Birefringence, Phys. Rev. Lett. 58, 1055 (1987).

[49] W. J. Munro and M. D. Reid, Transient macroscopic quantum superposition states in degenerate parametric oscillation using squeezed reservoir fields, Phys. Rev. A 52, 2388 (1995).

[50] Z. Wang, A. Marandi, K. Wen, R. L. Byer, and Y. Yamamoto, Coherent Ising machine based on degenerate optical parametric oscillators, Phys. Rev. A 88, 063853 (2013); A. Marandi, Z. Wang, K. Takata, R. L. Byer, and Y. Yamamoto, Network of time-multiplexed optical parametric oscillators as a coherent Ising machine, Nat. Photonics 8, 937 (2014); T. Inagaki, Y. Haribara, K. Igarashi, T. Sonobe, S. Tamate, T. Honjo, A. Marandi, P. L. McMahon, T. Umeki, K. Enbutsu, O. Tadanaga, H. Takenouchi, K. Aihara, K.-i. Kawarabayashi, K. Inoue, S. Utsunomiya, and H. Takesue, A coherent Ising machine for 2000-node optimization problems, Science 354, 603 (2016).

[51] S. E. Nigg, H. Paik, B. Vlastakis, G. Kirchmair, S. Shankar, L. Frunzio, M. H. Devoret, R. J. Schoelkopf, and S. M. Girvin, Black-Box Superconducting Circuit Quantization, Phys. Rev. Lett. 108, 240502 (2012); H. Goto, Z. Lin, T. Yamamoto, and Y. Nakamura, On-demand generation of traveling cat states using a parametric oscillator, Phys. Rev. A 99, 023838 (2019).

[52] W. Pfaff, C. J. Axline, L. D. Burkhart, U. Vool, P. Reinhold, L. Frunzio, L. Jiang, M. H. Devoret, and R. J. Schoelkopf, Controlled release of multiphoton quantum states from a microwave cavity memory, Nat. Phys. 13, 882 (2017).

[53] H. Goto, Bifurcation-based adiabatic quantum computation with a nonlinear oscillator network, Sci. Rep. 6, 21686 (2016); Universal quantum computation with a nonlinear oscillator network, Phys. Rev. A 93, 050301(R) (2016); S. Puri, C. K. Andersen, A. L. Grimsmo, and A. Blais, Quantum annealing with all-to-all connected nonlinear oscillators, Nat. Commun. 8, 15785 (2017).

[54] L. Luo, H. Liu, N. Huang, and Z. Wang, Speed-up coherent Ising machine with a squeezed feedback system, Opt. Express 28, 1914 (2020); D. Maruo, S. Utsunomiya, and Y. Yamamoto, Truncated Wigner theory of coherent Ising machines based on degenerate optical parametric oscillator network, Phys. Scr. 91, 083010 (2016); Y. Inui and Y. Yamamoto, Noise correlation and success probability in coherent Ising machines, arXiv:2009.10328v1.

[55] P. Drummond, K. McNeil, and D. Walls, Non-equilibrium transitions in sub/second harmonic generation, Opt. Acta 28, 211 (1981).

[56] L. Krippner, W. J. Munro, and M. D. Reid, Transient macroscopic quantum superposition states in degenerate parametric oscillation: Calculations in the large-quantum-noise limit using the positive $P$ representation, Phys. Rev. A 50, 4330 (1994).

[57] F.-X. Sun, Q. He, Q. Gong, R. Y. Teh, M. D. Reid, and P. D. Drummond, Discrete time symmetry breaking in quantum circuits: Exact solutions and tunneling, New J. Phys. 21, 093035 (2019).

[58] F.-X. Sun, Q. He, Q. Gong, R. Y. Teh, M. D. Reid, and P. D. Drummond, Schrödinger cat states and steady states in subharmonic generation with Kerr nonlinearities, Phys. Rev. A 100, 033827 (2019).

[59] R. Y. Teh, F.-X. Sun, R. E. S. Polkinghorne, Q. Y. He, Q. Gong, P. D. Drummond, and M. D. Reid, Dynamics of transient cat states in degenerate parametric oscillation with and without nonlinear Kerr interactions, Phys. Rev. A 101, 043807 (2020).

[60] F. Albarelli, M. G. Genoni, M. G. A. Paris, and A. Ferraro, Resource theory of quantum non-Gaussianity and Wigner negativity, Phys. Rev. A 98, 052350 (2018).

[61] H. Fearn and M. Collett, Representations of squeezed states with thermal noise, J. Mod. Opt. 35, 553 (1988).

[62] M. S. Kim, F. A. M. de Oliveira, and P. L. Knight, Properties of squeezed number states and squeezed thermal states, Phys. Rev. A 40, 2494 (1989).

[63] A. Gilchrist, P. Deuar, and M. D. Reid, Contradiction of quantum mechanics with local hidden variables for quadrature phase measurements on pair-coherent states and squeezed macroscopic superpositions of coherent states, Phys. Rev. A 60, 4259 (1999).

[64] S.-1. Ma, J.-k. Xie, and F.-1. Li, Generation of superposition coherent states of microwave fields via dissipation of a superconducting qubit with broken inversion symmetry, Phys. Rev. A 99, 022302 (2019).

[65] F. Fröwis, P. Sekatski, W. Dür, N. Gisin, and N. Sangouard, Macroscopic quantum states: Measures, fragility, and implementations, Rev. Mod. Phys. 90, 025004 (2018).

[66] A. Kenfack and K. Zyczkowski, Negativity of the Wigner function as an indicator of non-classicality, J. Opt. B: Quantum Semiclassical Opt. 6, 396 (2004).

[67] T. Baumgratz, M. Cramer, and M. B. Plenio, Quantifying Coherence, Phys. Rev. Lett. 113, 140401 (2014).

[68] G. Kryuchkyan and K. Kheruntsyan, Exact quantum theory of a parametrically driven dissipative anharmonic oscillator, Opt. Commun. 127, 230 (1996).

[69] C. W. Gardiner and M. J. Collett, Input and output in damped quantum systems: Quantum stochastic differential equations and the master equation, Phys. Rev. A 31, 3761 (1985).

[70] P. D. Drummond and M. Hillery, The Quantum Theory of Nonlinear Optics (Cambridge University Press, 2014).

[71] C. F. Ockeloen-Korppi, E. Damskägg, J.-M. Pirkkalainen, T. T. Heikkilä, F. Massel, and M. A. Sillanpää, Noiseless Quantum Measurement and Squeezing of Microwave Fields Utilizing Mechanical Vibrations, Phys. Rev. Lett. 118, 103601 (2017).

[72] P. Kinsler and P. D. Drummond, Quantum dynamics of the parametric oscillator, Phys. Rev. A 43, 6194 (1991).

[73] G. K. Brennen, An observable measure of entanglement for pure states of multi-qubit systems, Quantum Inf. Comput. 3, 619 (2003).

[74] E. G. Cavalcanti and M. D. Reid, Signatures For Generalized Macroscopic Superpositions, Phys. Rev. Lett. 97, 170405 (2006); Criteria for generalized macroscopic and mesoscopic quantum coherence, Phys. Rev. A 77, 062108 (2008); F. Fröwis, P. Sekatski, and W. Dür, Detecting Large Quantum Fisher Information with Finite Measurement Precision, Phys. Rev. Lett. 116, 090801 (2016); B. Yadin and V. Vedral, General framework for quantum macroscopicity in terms of coherence, Phys. Rev. A 93, 022122 (2016); M. D. Reid, Criteria to detect macroscopic quantum coherence, macroscopic quantum entanglement, and an Einstein-Podolsky-Rosen paradox for macroscopic superposition states, ibid. 100, 052118 (2019).

[75] K. E. Cahill and R. J. Glauber, Density operators and quasiprobability distributions, Phys. Rev. 177, 1882 (1969). 
[76] A. Pathak and J. Banerji, Wigner distribution, nonclassicality and decoherence of generalized and reciprocal binomial states, Phys. Lett. A 378, 117 (2014).

[77] Y.-C. Liang, Y.-H. Yeh, P. E. M. F. Mendonça, R. Y. Teh, M. D. Reid, and P. D. Drummond, Quantum fidelity measures for mixed states, Rep. Prog. Phys. 82, 076001 (2019).

[78] C.-W. Lee, J. Lee, H. Nha, and H. Jeong, Generating a Schrödinger-cat-like state via a coherent superposition of photonic operations, Phys. Rev. A 85, 063815 (2012). 